\title{
Hopf Bifurcation from Rotating Waves and Patterns in Physical Space*
}

\author{
Martin Golubitsky \\ Department of Mathematics \\ University of Houston \\ Houston, TX 77204-3476 \\ USA
}

\author{
Victor G. LeBlanc \\ Department of Mathematics \\ University of Ottawa \\ Ottawa, ON K1N 6N5 \\ CANADA
}

\author{
Ian Melbourne \\ Department of Mathematics \\ University of Houston \\ Houston, TX 77204-3476 \\ USA \\ January 13, 1998 \\ Revised version: November 2, 1999
}

\begin{abstract}
Hopf bifurcations from time periodic rotating waves to two frequency tori have been studied for a number of years by a variety of authors including Rand and Renardy. Rotating waves are solutions to partial differential equations where time evolution is the same as spatial rotation. Thus rotating waves can exist mathematically only in problems that have at least $\mathbf{S O}(2)$ symmetry. In this paper we study the effect on this Hopf bifurcation when the problem has more than $\mathbf{S O}(2)$ symmetry. These effects manifest themselves in physical space and not in phase space. We use as motivating examples the experiments of Gorman et al. on porous plug burner flames, of Swinney et al. on the Taylor-Couette system, and of a variety of people on meandering spiral
\end{abstract}

\footnotetext{
*In press: J. Nonlin. Sci.
} 
waves in the Belousov-Zhabotinsky reaction. In our analysis we recover and complete Rand's classification of modulated wavy vortices in the Taylor-Couette system.

It is both curious and intriguing that the spatial manifestations of the two frequency motions in each of these experiments is different and it is these differences that we seek to explain. In particular, we give a mathematical explanation of the differences between the nonuniform rotation of cellular flames in Gorman's experiments and the meandering of spiral waves in the Belousov-Zhabotinsky reaction.

Our approach is based on the center bundle construction of Krupa with compact group actions and its extension to noncompact group actions by Sandstede, Scheel, and Wulff.

\section{Introduction}

Rotating waves are time periodic solutions to differential equations where time evolution is the same as spatial rotation. It follows that rotating waves occur as solutions only in systems of differential equations having at least $\mathbf{S O}(2)$ symmetry, though, in general, rotating waves occur in experiments whose models contain more than $\mathbf{S O}(2)$ symmetry. In this paper we consider bifurcations from rotating waves to two frequency modulated rotating waves and make the point that the manifestation of the modulated waves in physical space depends crucially on the full symmetry group. To illustrate this point, we introduce three experiments where rotating and modulated rotating waves are observed. We then describe the symmetry groups for models of these experiments and the types of modulated waves that are observed in each experiment. We end this introduction with a description of the results that we have obtained and how they bear on the three experiments.

\section{Rotating Waves in Experiments}

Rotating waves have been observed in a variety of experiments - both physical and numerical. In particular, rotating waves occur in the Taylor-Couette system as wavy vortices (see Andereck et al. [1] and Figure 2), in the Belousov-Zhabotinsky chemical reaction as spiral waves (see Winfree [41] and Figure 3), and in laminar premixed flames as cellular patterns (Gorman et al. [19] and Figure 1). Each of these rotating waves has also been observed numerically as a patterned solution to PDE models for the corresponding experiments: rotating waves have been observed in the Navier-Stokes equations modeling the Taylor-Couette system [31], in reaction-diffusion equations loosely modeling BZ reactions $[2,26]$, and in reaction-diffusion models loosely modeling combustion [5, 23]. 

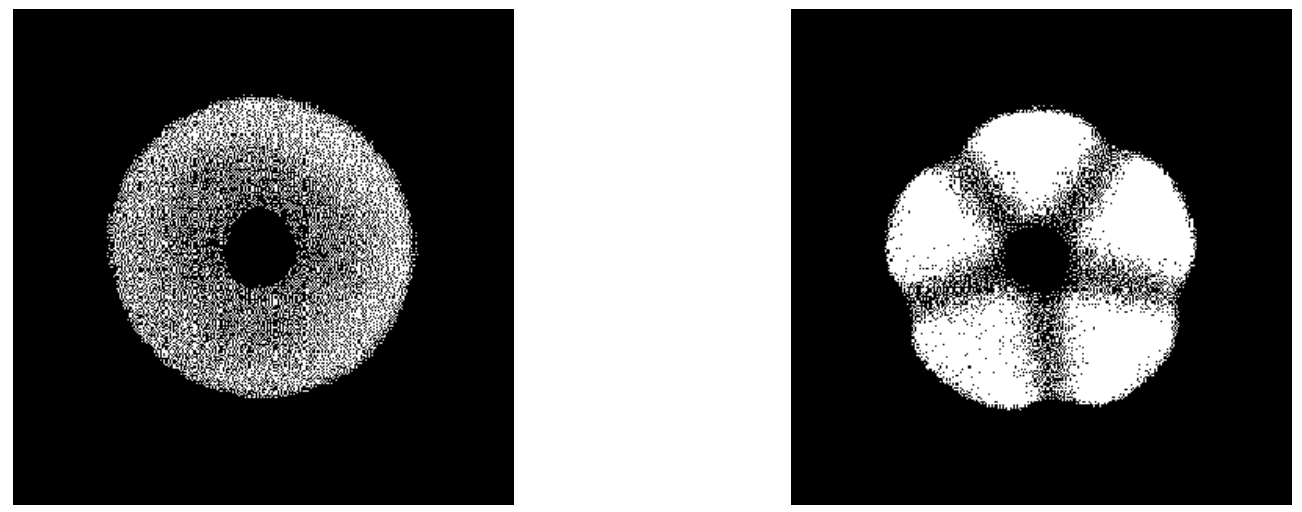

Figure 1: Flames on a circular burner. (Left) A circularly symmetric flame. (Right) A steady five-cell flame with $\mathbf{D}_{5}$ symmetry. Images courtesy of M. Gorman.

\section{Symmetries in the Experiments}

Since we view symmetry as a modeling assumption, it is important to understand how the symmetry group $\Gamma$ for models of each of these experiments is determined. Some of the symmetries are clear, being based on the geometry of the apparatus and the homogeneity of the experiment; other symmetries are less transparent.

For example, Gorman's laminar premixed flame experiment is performed on a circular burner and is modeled by $\Gamma=\mathbf{O}(2)$ symmetry. Moreover, the transitions that are observed in this experiment are consistent with the assumption of $\mathbf{O}(2)$ symmetry, in the following sense. It is well known that steady-state bifurcation from an invariant equilibrium with $\mathbf{O}(2)$ symmetry on a circular domain leads to equilibria having a cellular structure and that Hopf bifurcation leads to standing and rotating waves [14]. All of these states are observed in the flame experiment, and the cellular structures and the standing waves are observed as direct transitions from a circularly symmetric flame [16].

The geometry is not always sufficient to detect all of the relevant symmetries. In the Taylor-Couette experiment, the cylindrical geometry leads to the assumption of $\Gamma=\mathbf{S O}(2) \times$ $\mathbf{Z}_{2}$ symmetry. As Taylor [40] noted, the existence of Taylor vortices bifurcating from Couette flow argues for the assumption of periodic boundary conditions in the axial direction (see Figure 2); that is, the assumption of $\mathbf{S O}(2) \times \mathbf{O}(2)$ symmetry in the experiment. Moreover, this symmetry is consistent with the observed Hopf bifurcation from Couette flow to spiral vortices [8, 15].

In the BZ reaction, the situation is even more complicated. It seems best to model these experiments by ignoring lateral boundaries. Barkley's analysis [3] of resonant linear drift of spiral waves in this experiment makes a compelling case for the assumption of full 

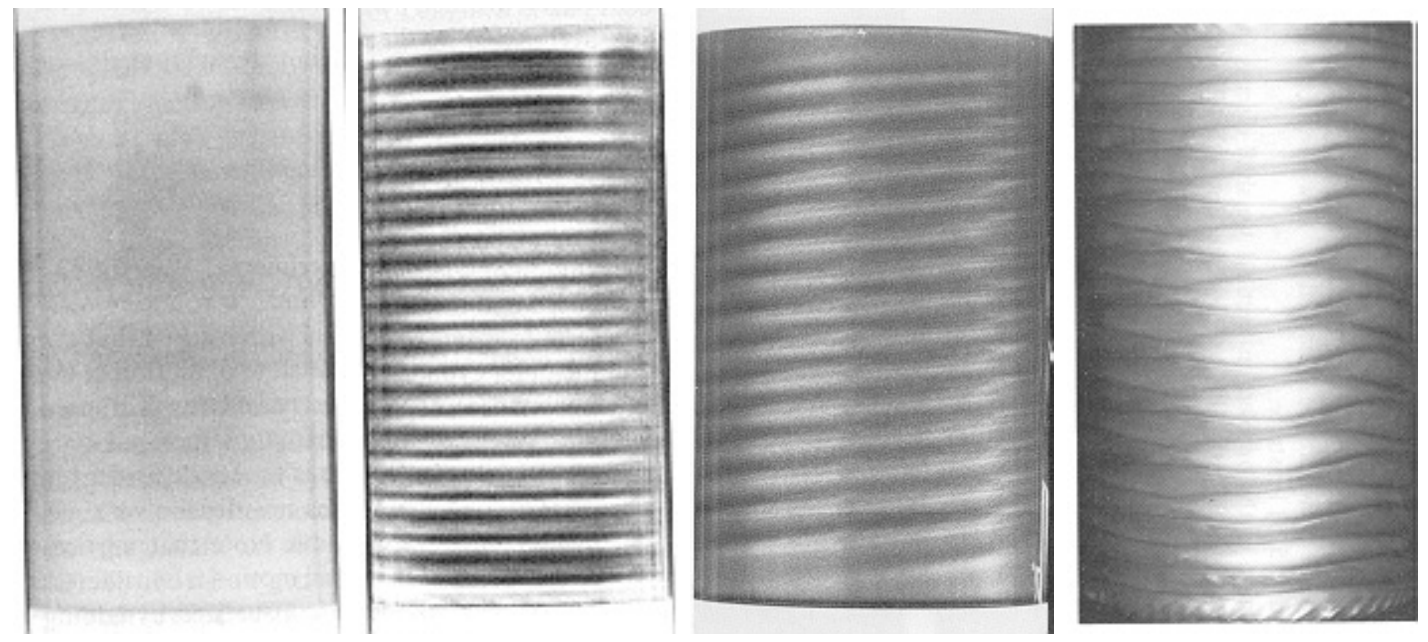

Figure 2: Taylor-Couette Experiment. From left to right: Couette flow, Taylor vortices, spiral vortices, and wavy vortices. Pictures courtesy of H.L. Swinney.

Euclidean $\Gamma=\mathbf{E}(2)$ symmetry including translations. Moreover, the mathematical results in $[42,37,38,13]$ support this assumption.

The rotating waves in each of these experiments have cyclic symmetry for the instantaneous pattern. We denote the symmetry group (or isotropy subgroup) of the pattern at a given instant in time by $\Sigma_{\text {rot }}$. The cellular flame pattern has $k$ cells (see Figure 1), and a spiral can have $k$ arms - though one-armed spirals are what is usually observed in BZ reactions (see Figure 3). See [34] and references therein for experimental observations of multi-armed spirals $(k \geq 2)$. For both flames and spirals, $\Sigma_{\text {rot }}=\mathbf{Z}_{k}$. The wavy vortices (see Figure 2) have an azimuthal wave number $k$, and an additional symmetry - flip up and down coupled with a half wave length azimuthal rotation (a glide reflection symmetry). The square of this symmetry is the generator of the pure azimuthal rotation symmetry so the isotropy subgroup of wavy vortices is $\mathbf{Z}_{2 k}$. In Table 1 we present the relevant group theoretic data for each experiment.

\begin{tabular}{|c|c|c|c|}
\hline Experiment & Rotating Wave & $\Gamma$ & $\Sigma_{\text {rot }}$ \\
\hline \hline Taylor-Couette & wavy vortices & $\mathbf{S O}(2) \times \mathbf{O}(2)$ & $\mathbf{Z}_{2 k}$ \\
\hline flames & rotating cells & $\mathbf{O}(2)$ & $\mathbf{Z}_{k}$ \\
\hline BZ reaction & 1-armed spirals & $\mathbf{E}(2)$ & $\mathbf{1}$ \\
\hline
\end{tabular}

Table 1: Symmetry data for rotating waves. 

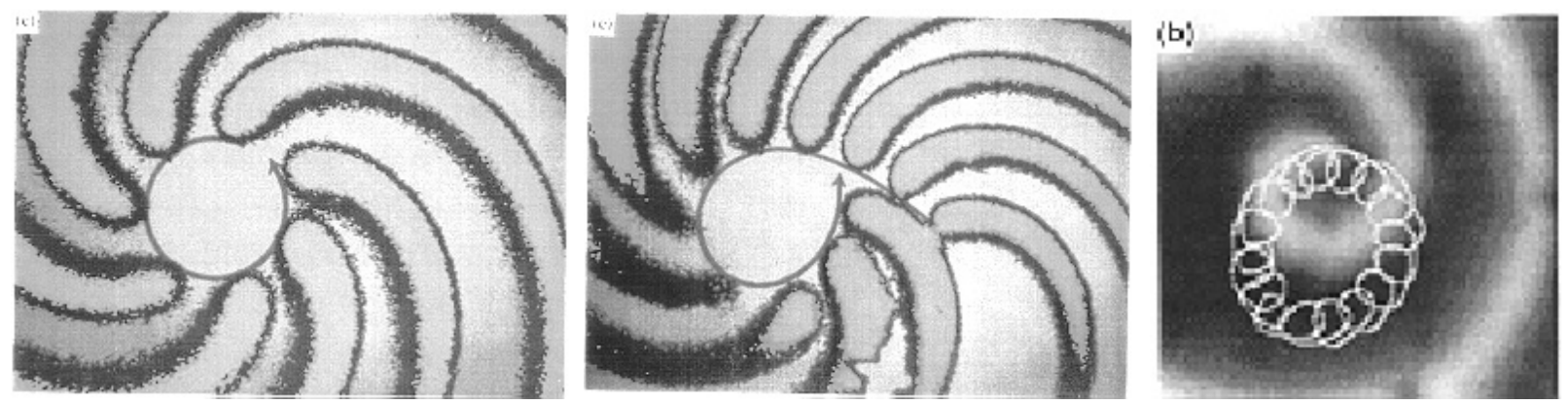

Figure 3: Belousov-Zhabotinsky Experiment. (Left) spiral waves with seven superimposed images showing the tip traversing a circle (from [39]). (Center) meandering spiral waves with eleven superimposed images showing the tip beginning to meander (from [39]). (Right) Single image of a meandering spiral wave with superimposed tip trajectory showing a petals in flower pattern (from [29]). Pictures courtesy of H.L. Swinney.

\section{Hopf Bifurcations to Quasiperiodic Motions}

In each of these experiments, Hopf bifurcation from rotating waves to a quasiperiodic motion, or modulated rotating wave, has been observed. In the Taylor-Couette system, wavy vortices bifurcate to modulated wavy vortices [20, 21, 9]; in the BZ reaction, spiral waves begin to meander quasiperiodically (see Figure 3) and even linearly drift [44, 45, 30, 24, 25, 39, 29]; and in laminar premixed flames, the cellular pattern appears to rotate rigidly but with an angle of rotation that depends quasiperiodically on time [19, 6, 7, 33]. In each of these experiments, the basic Hopf bifurcation from a single frequency time periodic rotating wave to quasiperiodic motion in phase space is now understood [22, 4, 7]. Even the resonant Hopf bifurcation to linear drifting spiral waves is understood [3, 42, 38, 13].

Hopf bifurcations from rotating waves to modulated rotating waves have been studied by a number of authors including Rand [35] and Renardy [36]. In addition, Rand classifies the various types of modulated waves that occur in systems with circular symmetry. This classification applies directly to the flame experiment (as pointed out in [7]) and less directly to the Taylor-Couette experiment (since wavy vortices have an additional glide reflection symmetry which is not taken into account in [35]). It is straightforward, as we show in Section 6, to complete Rand's classification of modulated wavy vortices to include the glide reflection symmetry. This extended classification accounts for additional states that have been observed in more recent experiments [43, 9].

What is missing is an understanding of the way in which these modulated waves manifest themselves in physical space. In this paper we use symmetry arguments to show why cellular flames appear to rotate rigidly but nonuniformly, and why spirals exhibit flower-like 
meandering in the BZ reaction (see Figure 3). The classification of Rand is driven by the symmetry group $\Sigma_{\text {rot }}$ of the rotating wave state. In contrast, we show that the motion of the pattern in physical space depends on both the symmetry $\Sigma_{\text {rot }}$ of the rotating wave and the full symmetry group $\Gamma$ of the experiment.

\section{A Definition of Spatial Pattern}

In both the physical and numerical experiments, patterns are formed as follows. Let $U(X, t)$ be a solution to a partial differential equation or the state of an experiment where $X$ is in some physical domain $\mathcal{D}$ and $t$ is time. For example, in the Taylor-Couette system, $U$ consists of the three velocity components of the fluid and the pressure variable, and $\mathcal{D} \subset \mathbf{R}^{3}$ is the region between the concentric cylinders. When we view the Taylor-Couette experiment, we look at an observable scalar quantity $u(x, t)$ which is the intensity of light reflected off of silver platelets in the fluid; here $x$ lies in the surface of the outer cylinder, which we denote by $\Omega$. We call $u$ an observable of the state $U$. For observables to be useful as a vehicle for understanding pattern, the transformation from $U$ to $u$ must be continuous and T-equivariant.

Similarly, in the BZ reaction $u$ is the concentration of an active chemical and $\Omega=\mathbf{R}^{2}$; while in the flame experiment $u$ is the intensity of light (or heat) produced by the flame and $\Omega \subset \mathbf{R}^{2}$ is a circular disk. In PDE systems, $u$ is some function of the solution vector perhaps one of its components.

We define a pattern at time $t$ to be the region in physical space

$$
\mathcal{P}(t)=\{x \in \Omega: u(x, t) \geq c\}
$$

for some fixed scalar $c$. For example, in the BZ reaction, the pattern is the region where the observed color is red (or blue). This region consists of those points in the petri dish where a chemical concentration is larger than some critical concentration.

Note that patterns associated to rotating wave solutions have a particularly elementary structure

$$
\mathcal{P}(t)=R_{t} \mathcal{P}(0)
$$

where $R_{t}$ is rotation through angle $t$ (in appropriate units). So, for example, a spiral wave is a pattern in the concentration of a fixed chemical in physical space that rigidly rotates at constant speed as time evolves.

On bifurcation to quasiperiodic motion, the change in the pattern in time has two components: shape change in the pattern and rigid motion of the pattern corresponding to the symmetry group of the experiment. It is our contention that in Hopf bifurcation from a 
rotating wave the shape change is less important to the observed pattern evolution than is the change in the rigid motion. To make this point precise, we introduce the notions of inner and outer patterns.

\section{Inner and Outer Patterns}

Suppose that $\mathcal{P}(t)$ is the pattern of a modulated rotating wave. As we explain in Section 2, we can bound the temporal fluctuation of $\mathcal{P}(t)$ in terms of an inner pattern $\mathcal{Q}^{\text {inner }}$ and an outer pattern $\mathcal{Q}^{\text {outer }}$, where

$$
\mathcal{Q}^{\text {inner }} \subset \mathcal{P}(0) \subset \mathcal{Q}^{\text {outer }}
$$

These time independent bounds have two important properties. First, as the bifurcation parameter approaches the point of Hopf bifurcation $\mathcal{Q}^{\text {inner }}$ and $\mathcal{Q}^{\text {outer }}$ limit on the (instantaneous time) pattern associated to the rotating wave. Second, for every time $t$, there is a group element $\gamma_{t} \in \Gamma$ such that $\gamma_{t}$ depends smoothly on $t, \gamma_{0}=e$, and

$$
\gamma_{t}\left(\mathcal{Q}^{\text {inner }}\right) \subset \mathcal{P}(t) \subset \gamma_{t}\left(\mathcal{Q}^{\text {outer }}\right)
$$

For example, when $\Gamma=\mathbf{O}(2)$ the pattern associated to the modulated wave is bounded between two patterns that rotate rigidly with nonuniform speed. Within a small error, the modulated wave pattern itself appears to rotate rigidly with nonuniform speed, the error due to the shape change being bounded between the inner and outer patterns. See Figure 4. The corresponding patterns when $\Gamma=\mathbf{E}(2)$ are shown in Figure 5. In particular, note that the spiral patterns in that figure do not have a fixed center of rotation.

\section{Center Bundles}

As shown in Section 2 the formulation of inner and outer patterns relies on the mathematical framework of center bundles. Center bundles were introduced by Krupa [27] in the analysis of bifurcations from relative equilibria for ODEs with compact symmetry group $\Gamma$. The theory was generalized by Sandstede et al. [37, 38] to include PDEs with noncompact symmetry groups. In particular, their theory applies to the nonsmooth representations that occur, for example, in reaction diffusion equations with Euclidean symmetry.

The geometric idea behind the center bundle is that when a relative equilibrium loses stability there are a set of critical directions at each point on the group orbit of the relative equilibrium. These unstable directions form a bundle over the group orbit: the center bundle. The unstable directions correspond to changes in pattern shape in physical space while the group orbit directions correspond to rigid motions of the pattern. 

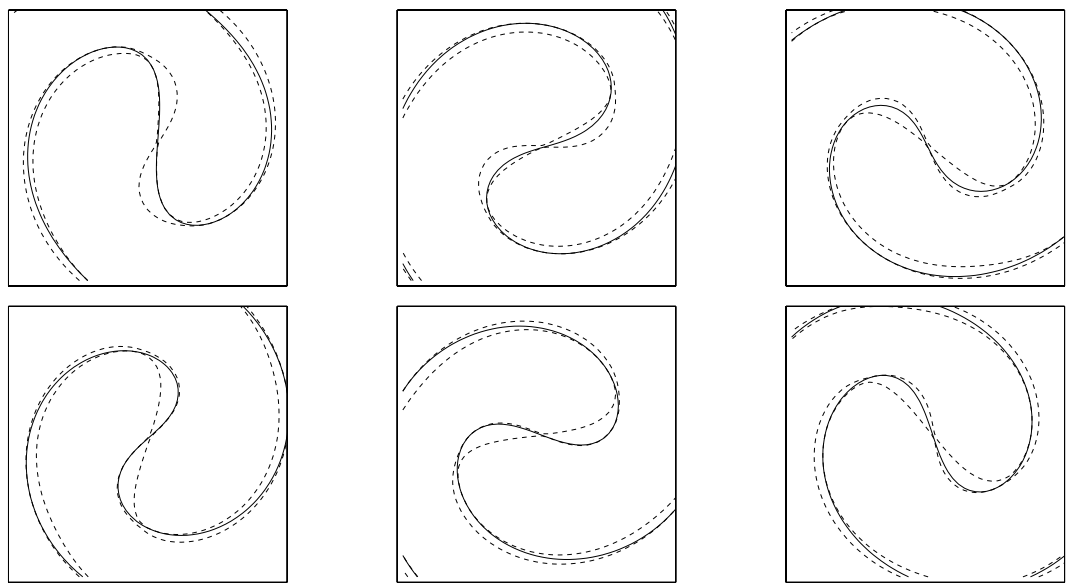

Figure 4: Inner and outer patterns for a nonuniformly rotating spiral wave in a circular domain $(\Gamma=\mathbf{O}(2))$. The dashed contours show the boundaries of the inner and outer patterns. The six snapshots show these contours rotating rigidly (but with nonuniform speed). The actual pattern (solid contour) is trapped between these boundaries. The snapshots show how the additional fluctuations in the shape change of the actual pattern are limited by the inner and outer patterns.
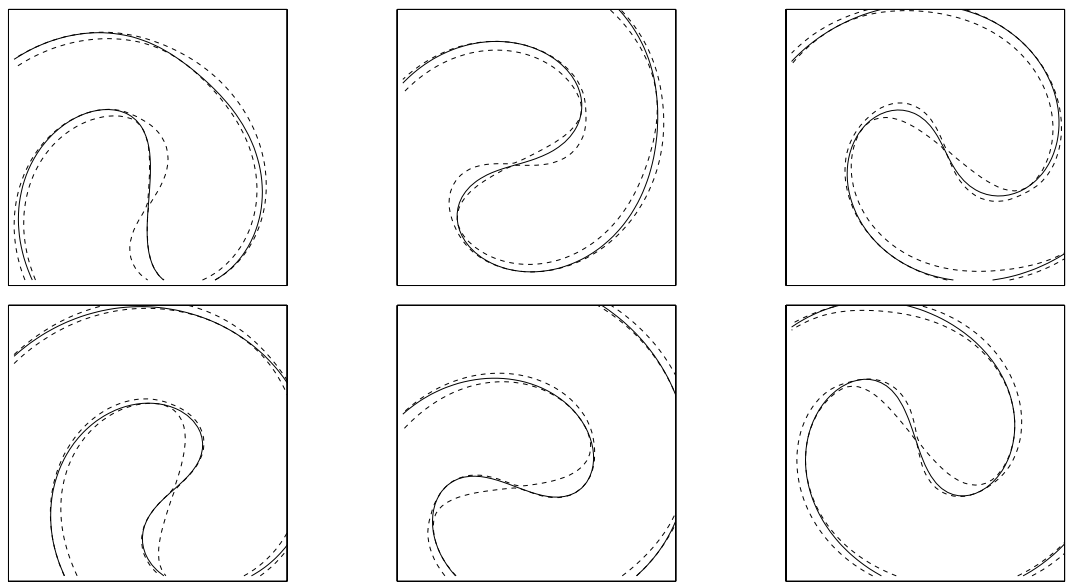

Figure 5: Inner and outer patterns for a meandering spiral wave in the plane $(\Gamma=\mathbf{E}(2))$. The interpretation of the Figure is the same as for Figure 4. However, the inner and outer patterns now rigidly meander (rotate with a moving center of rotation). 
The equations on the center bundle are often difficult to solve directly. Fiedler et al. [11] show how to lift the center bundle equations over the group orbit to skew product equations on the group $\Gamma$ itself, which are often easier to solve.

\section{Tip Evolution Using Fourier-Bessel Functions}

As described above, the arguments in this paper apply to the full patterned state in physical space. However, to obtain a more convenient graphical representation of such results, it is standard to plot the time evolution of some distinguished marker on the pattern (such as the 'tip' of a spiral). Figure 6 is computed numerically in this manner using spiral-like Fourier-Bessel functions, see Section 7 for details.

The time evolution of a rotating wave is shown in Figure 6(a). In this case, the 'tip' traces out a circle with uniform speed. Figure 6(b) corresponds to Figure 4 and shows the behavior of a modulated rotating wave in a circular domain. The tip no longer traces out a circle, but the radial variation is negligible. The approximate circle is traced out with nonuniform periodically varying speed, as shown in Figure 7.

In contrast, Figure 6(c) (which corresponds to Figure 5) depicts the modulated rotating wave of Figure 6(b) but in an infinite planar domain. The radial excursions are now appreciable and correspond to the phenomenon known as meander. This supports our assertion that quasiperiodic meander (even away from resonance) requires Euclidean symmetry without which the motion would resemble the nonuniform rotation that occurs in the flame experiment Figure 6(b). More precisely, what distinguishes meandering from nonuniform rotation is the quasiperiodic translation of the pattern, coupled with the quasiperiodic rotation of the pattern.

Figure 6(d)-(f) show the modulated rotating wave in an infinite planar domain close to resonance. The quasiperiodic translations are now of sufficiently large magnitude that welldefined petals are observed close to the onset of the modulated rotating wave. Note that in contrast to (c), in (d)-(f) it is the petals that are of roughly the same size as the circle in (a). When the petals change from inwards (d) to outwards (e) — as a second parameter is varied - the spiral tip appears to make circular excursions which drift linearly to infinity (f) $[44,45,3]$. This resonance phenomenon has been seen in numerical solutions to reaction diffusion systems [2] and in chemical experiments [29]. 


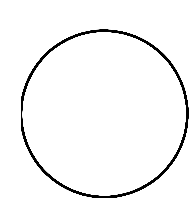

(a)

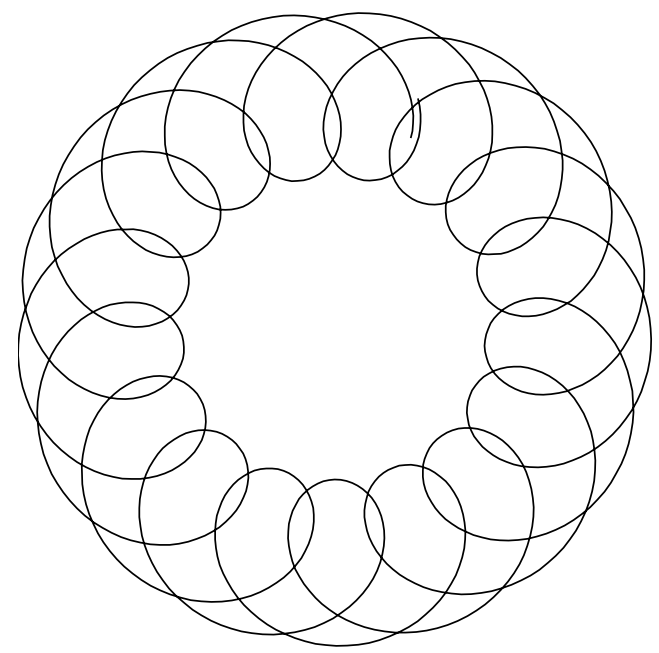

(d)

(b)
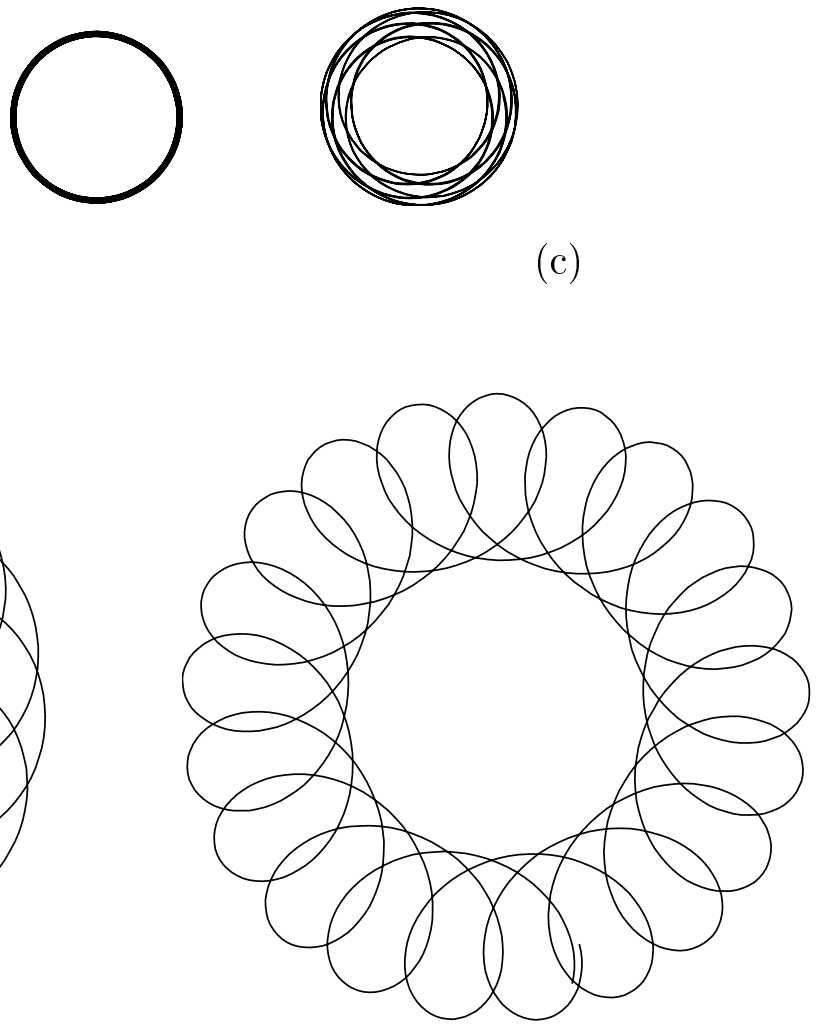

(e)

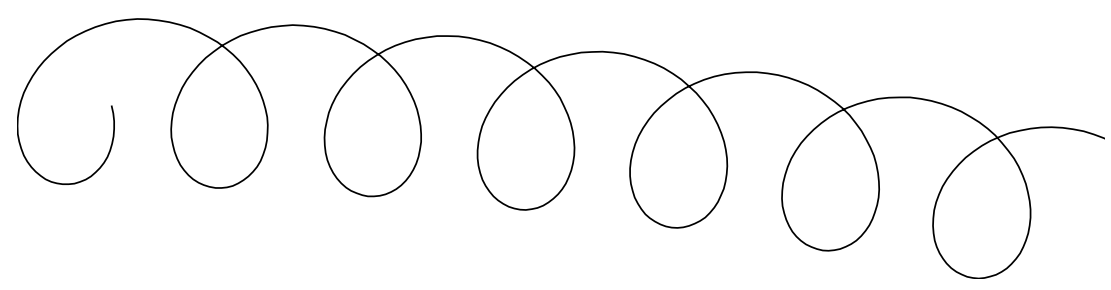

(f)

Figure 6: (a) Uniform rotation, (b) Nonuniform rotation, (c) Meandering (away from resonance), (d) Meandering near resonance (petals inwards), (e) Meandering near resonance (petals outwards), (f) Linear drift 


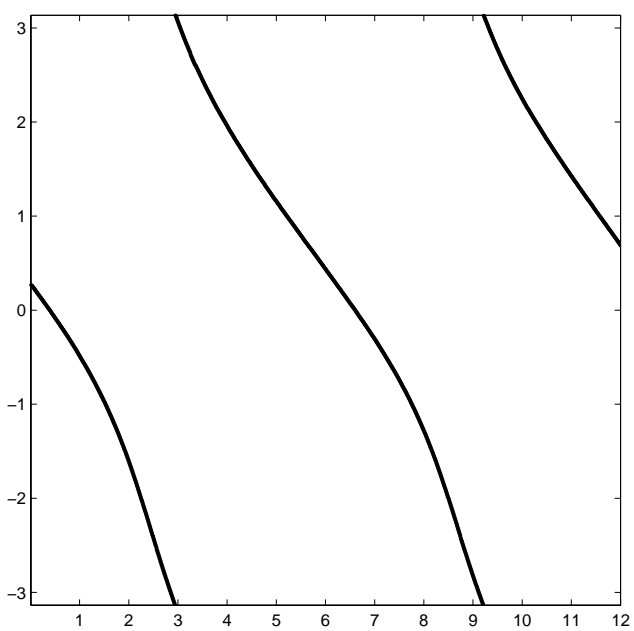

Figure 7: Nonuniformity of the rotation of the modulated rotating wave in Figure 6(b). The graph shows angle plotted as a function of time

The remainder of the paper is organized as follows. The notions of inner and outer pattern are formalized in Section 2. In Section 3, we analyze Hopf bifurcation from rotating waves with trivial isotropy $\left(\Sigma_{\text {rot }}=1\right.$ ) in systems with $\mathbf{O}(2)$ and $\mathbf{E}(2)$ symmetry. We pay particular attention to the contrasting behavior of nonuniform rotation (flames) and meander (spirals) in physical space. The corresponding bifurcations for states with nontrivial isotropy $\left(\Sigma_{\text {rot }}=\mathbf{Z}_{k}\right)$ are analyzed in Sections 4 and 5 respectively. In addition, we describe in Section 4 how the "reversing states" of Landsberg and Knobloch [28] fit into our analysis, and we describe a scenario which may explain the "ratcheting states" observed in the flames experiment. Bifurcation to modulated wavy vortices in the Taylor-Couette system $(\mathbf{S O}(2) \times$ $\mathbf{O}(2)$ symmetry) is analyzed in Section 6 . The details for the numerically generated figures in this paper are given in Section 7. Some of the more tedious proofs are postponed until Section 8.

\section{Inner and Outer Patterns}

Suppose that $\Gamma$ acts on some physical domain $\Omega \subset \mathbf{R}^{n}$ where $n=2$ or $n=3$. Let $\mathcal{H}$ be a space of observables $u: \Omega \rightarrow \mathbf{R}$ corresponding to states $U$ of an experiment or solutions $U$ to a system of differential equations. We assume that $\Gamma$ acts on $\mathcal{H}$ by

$$
\gamma u(x)=u\left(\gamma^{-1} x\right)
$$

where $u \in \mathcal{H}$ and $\gamma \in \Gamma$. 
We define the pattern associated to a function $u \in \mathcal{H}$ to be the region in physical space

$$
\mathcal{P}_{u}=\{x \in \Omega: u(x) \geq c\}
$$

for some fixed real number $c$. Note that the patterns associated to $\gamma u$ and $u$ are related by $\gamma$, that is,

$$
P_{\gamma u}=\gamma P_{u}
$$

For a time dependent function $u(\cdot, t) \in \mathcal{H}$, define the pattern at time $t$ in an analogous way:

$$
\mathcal{P}_{u}(t)=\{x \in \Omega: u(x, t) \geq c\} .
$$

For example, suppose that $u$ is the observable of a rotating wave; that is,

$$
u(x, t)=R_{t} u_{0}(x)
$$

where $u_{0}(x)=u(x, 0)$. It follows from $(2.2)$ that

$$
\mathcal{P}_{u}(t)=R_{t} \mathcal{P}_{u}(0)
$$

that is, the pattern associated to the rotating wave rigidly rotates in space with constant speed.

\section{The Pattern of a Modulated Rotating Wave}

Let

$$
v(x, t)=R_{t} v_{0}(x)
$$

be an observable of a rotating wave solution $V(x, t)=R_{t} V_{0}(x)$ with isotropy subgroup $\Sigma_{\text {rot }}=\Sigma_{V_{0}}$ to the PDE

$$
U_{t}=\mathcal{F}(U)
$$

The center bundle theory (Krupa [27] for $\Gamma$ compact and Sandstede et al. [37, 38] for noncompact $\Gamma$ ) shows that in a neighborhood of this rotating wave the vector field $\mathcal{F}$ has the decomposition

$$
\mathcal{F}=\mathcal{F}_{N}+\mathcal{F}_{T}
$$

where $\mathcal{F}_{T}$ is tangent to group orbits, $\mathcal{F}_{N}$ is transverse to group orbits, and both vector fields are $\Gamma$-equivariant. Moreover, if we let $N_{0}$ be the normal section (in phase space) to the 
group orbit at $V_{0}$, then $\mathcal{F}_{N}$ restricts to $g: N_{0} \rightarrow N_{0}$ where $g\left(V_{0}\right)=0$. Note that $g$ is a $\Sigma_{\text {rot }}$ equivariant vector field on $N_{0}$.

One result of the center bundle construction is that in a neighborhood of a rotating wave, all solutions can be written as

$$
U(X, t)=\gamma(t) Y(X, t)
$$

where $\gamma(t) \in \Gamma$ is a smooth curve and $Y(x, t) \in N_{0}$ is a solution to the normal vector field equation

$$
Y_{t}=g(Y)
$$

Therefore, Hopf bifurcation from a rotating wave reduces to a Hopf bifurcation from an equilibrium in (2.4) coupled with a drift along group orbits. The fact that the transformation from states to observables is assumed to be $\Gamma$-equivariant leads to the identity

$$
u(x, t)=\gamma(t) y(x, t),
$$

where $u$ is the observable of $U$ and $y$ is the observable of $Y$.

Suppose now that $y(x, t)$ is time periodic with corresponding modulated rotating wave solution $u(x, t)=\gamma(t) y(x, t)$, as in (2.5). We obtain a time periodic pattern (originating from the normal equations):

$$
\mathcal{P}_{y}(t)=\{x \in \Omega: y(x, t) \geq c\} .
$$

Definition 2.1 The inner pattern associated to the modulated rotating wave $u$ is the intersection

$$
\mathcal{Q}^{\text {inner }}=\bigcap_{t} \mathcal{P}_{y}(t)=\left\{x \in \Omega: \min _{t} y(x, t) \geq c\right\} .
$$

The outer pattern associated to $u$ is the union

$$
\mathcal{Q}^{\text {outer }}=\bigcup_{t} \mathcal{P}_{y}(t)=\left\{x \in \Omega: \max _{t} y(x, t) \geq c\right\} .
$$

It follows immediately from Definition 2.1 that for every $t$

$$
\mathcal{Q}^{\text {inner }} \subset \mathcal{P}_{y}(t) \subset \mathcal{Q}^{\text {outer }} \text {. }
$$

Identity (2.2) implies that the patterns for the modulated rotating wave $u$ satisfy

$$
\mathcal{P}_{u}(t)=\gamma(t) \mathcal{P}_{y}(t)
$$

from which it follows that

$$
\gamma(t) \mathcal{Q}^{\text {inner }} \subset \mathcal{P}_{u}(t) \subset \gamma(t) \mathcal{Q}^{\text {outer }}
$$

Thus, the pattern of the modulated rotating wave evolves in time, bounded by a time dependent rigid motion of the region between $\mathcal{Q}^{\text {inner }}$ and $\mathcal{Q}^{\text {outer }}$. 


\section{The Dependence of Pattern on the Bifurcation Parameter}

In the preceding discussion we suppressed the dependence of the modulated wave on the bifurcation parameter $\lambda$. We now note that when $\lambda$ is near the bifurcation point, then $\mathcal{Q}^{\text {inner }}$ and $\mathcal{Q}^{\text {outer }}$ will be approximately equal to $\mathcal{P}_{v}(0)$ - the pattern of the rotating wave at a fixed moment in time. Indeed, these sets are all equal at the bifurcation point of $\lambda$ as $y=v_{0}$ there.

To see that the visible pattern associated with the modulated wave is virtually identical to the visible pattern of the rotating wave at an instant in time, we need one final assumption. We need to assume that the level contour $\left\{x \in \Omega: v_{0}(x)=c\right\}$ is a manifold. If not, the pattern can undergo a bifurcation just due to the observation process. Note that this last assumption is valid generically.

\section{Nonuniform Rotations and Meandering}

In the flame experiments and in the model equations, the bifurcation from the cellular rotating wave produces a state that appears to rigidly rotate with speed varying periodically in time (so that there are two independent frequencies). Palacios et al. [33] call this a "nonuniformly rotating" state. Indeed, such a state cannot be exactly described this way because a solution to a differential equation that lies exactly in a group orbit (in this case the group orbit given by the rotation subgroup) must produce linear flow along the group orbit, that is, the speed of rotation must be constant. In fact, careful observation of this state shows [33] that the cellular pattern does vary periodically in time — but by a small amount.

In contrast, the corresponding bifurcation for spirals in the BZ reaction leads to quasiperiodic meandering where the spiral pattern rotates and translates rigidly in space. Again, there is a small periodic fluctuation in the shape of the spiral.

In phase space, these modulated rotating waves are indistinguishable quasiperiodic motions, but their behavior in physical space, nonuniform rigid rotation and meandering, are strikingly different. We show that this behavior can be explained using center bundle reduction and the notion of inner and outer patterns. In particular, the quasiperiodic variation decouples into a global rigid motion (along group orbits) and a local fluctuation that is bounded by the inner and outer patterns.

For simplicity of exposition, we restrict in this section to bifurcations from rotating waves with no spatial symmetry, that is, we assume $\Sigma_{\text {rot }}=\mathbf{1}$. In circular domains, we obtain modulated rotating waves that rotate almost rigidly but with nonuniform speed. In unbounded planar domains, we obtain modulated rotating waves that meander. 
We remark that by ignoring translation symmetry, the work of [4] does not fully explain the transition to quasiperiodic meandering of one-armed spirals. In a circular domain, Hopf bifurcation from a rotating wave spiral solution leads to spirals that vary quasiperiodically in time, but does not lead directly to the flower patterns characteristic of meander. (Compare Figures 6(b) and 6(c), also Figures 4 and 5.) This additional structure arises from the Euclidean symmetry in the infinite plane.

\section{Nonuniform Rotation in Circular Domains}

In circular domains, the symmetry group is $\Gamma=\mathbf{S O}(2)$ or $\Gamma=\mathbf{O}(2)$; the analysis of these two cases is identical. Let $X$ be a rotating wave, so $X$ is simultaneously an $\mathbf{S O}(2)$ group orbit and a periodic orbit. We assume that the points in $X$ have trivial isotropy.

In the event of Hopf bifurcation, there is a three-dimensional center bundle and this is a trivial bundle $X=S^{1} \times \mathbf{C}$ by [13]. We choose coordinates $(\varphi, q)$ where $\varphi \in S^{1}$ measures the angle of rotation along the $\mathbf{S O}(2)$ group orbit and $q \in \mathbf{C}$ measures the deviation of the modulated rotating wave pattern from the basic rotating wave pattern. We shall refer to $q$ as the shape of the modulated rotating wave.

The action of an element $\theta \in \mathbf{S O}(2)$ on $(\varphi, q) \in X$ is given by

$$
\theta \cdot(\varphi, q)=(\varphi+\theta, q)
$$

and $\mathbf{S O}(2)$ equivariance leads to equations on the center bundle of the form [13]

$$
\begin{aligned}
\dot{\varphi} & =F^{\varphi}(q, \lambda), \\
\dot{q} & =F^{q}(q, \lambda) .
\end{aligned}
$$

Let $\omega_{\text {rot }}$ and $\omega_{\text {bif }}$ be the frequencies for the rotating wave and the Hopf bifurcation respectively. So $F^{\varphi}(0,0)=\omega_{\text {rot }}$ and $\left(d F^{q}\right)_{0,0}=i \omega_{\text {bif. }}$. We claim that solutions to these equations satisfy

$$
\begin{aligned}
\varphi(t, \lambda) & =\omega_{\text {rot }}(\lambda) t+\sqrt{\lambda} \psi(t, \lambda), \\
q(t, \lambda) & =\sqrt{\lambda} q_{0}(t, \lambda)
\end{aligned}
$$

where $q_{0}(t, \lambda)$ and $\psi(t, \lambda)$ are $2 \pi / \omega_{\text {bif }}(\lambda)$ periodic, $\omega_{\text {bif }}(0)=\omega_{\text {bif }}$ and $\omega_{\text {rot }}(0)=\omega_{\text {rot }}$.

To verify (3.1) and (3.2) solve the $\dot{q}$ equation directly obtaining a branch of $2 \pi / \omega_{\text {bif }}(\lambda)$ periodic solutions $q(t, \lambda)$ and then substitute (3.2) into the $\dot{\varphi}$ equation. We obtain a $2 \pi / \omega_{\text {bif }}(\lambda)$ periodic vector field $F^{\varphi}(q(t, \lambda), \lambda)$ with zeroth Fourier coefficient

$$
\omega_{\text {rot }}(\lambda)=\frac{\omega_{\text {bif }}(\lambda)}{2 \pi} \int_{0}^{2 \pi / \omega_{\text {bif }}(\lambda)} F^{\varphi}(q(t, \lambda), \lambda) d t .
$$

In particular, $\omega_{\text {rot }}(0)=F^{\varphi}(0,0)=\omega_{\text {rot }}$. Integrating the vector field $F^{\varphi}(q(t, \lambda), \lambda)$, we obtain (3.1) where $\psi(t, \lambda)$ is $2 \pi / \omega_{\mathrm{bif}}(\lambda)$ periodic. 


\section{Implications of (3.1) and (3.2) for Patterns in Physical Space}

The pattern approximately rigidly rotates with approximate speed $\omega_{\text {rot }}$ but speeds up and slows down with approximate frequency $\omega_{\text {bif }}$.

More precisely, if the shape change $q(t, \lambda)$ is regarded as negligible, then the time evolution is rigid rotation through angle $\varphi(t, \lambda)$. Since the frequencies $\omega_{\text {bif }}(\lambda)$ and $\omega_{\text {rot }}(\lambda)$ are typically independent, the time-dependence is quasiperiodic, hence the rigid rotation is nonuniform. For $\lambda$ small, the speed is approximately $\omega_{\text {rot }}$ and the second frequency enters as a small amplitude modulation of the basic frequency.

As already mentioned, the shape change $q(t, \lambda)$ is necessarily nonzero. Moreover, $\psi$ and $q$ are each of order $\sqrt{\lambda}$ and hence might seem to be of equal significance for the phenomena in physical space. Nevertheless, it is our contention that the $\psi$-effect outweighs the $q$-effect, and that the behavior of the modulated rotating wave in physical space is to a first approximation as described above. Our reasoning is that the drift along the group orbit is an organized controlled effect that can be analyzed as we have done above. Moreover, it results in a rigid motion of the pattern globally in physical space. The notion of inner and outer pattern formalizes this idea, see Figure 4. In contrast, the shape change is a somewhat arbitrary disorganized localized effect.

Further explanation of the subordinate role of shape change is possible on the grounds that often the eigenfunctions that determine the shape change have steep vertical fronts (as in spiral waves); so shape change (idealized as a change in a level contour) has little effect in the planar directions. (Indeed, spiral waves are sometimes modeled as having infinitely steep fronts, in which case our discussion is exact.) However, such steepness does not appear to be required in practice. Figure $6(\mathrm{~b})$ was produced with smooth eigenfunctions and is at the same scale as the remaining diagrams in Figure 6. The arbitrary shape change could in principle produce a radial fluctuation even in Figure 6(b) and could in principle cancel out the radial fluctuation of Figure 6(c) - but this is not likely in practice. (A movie with time $t$ varying is more convincing, since it allows the visual distinction between global variation (drift) and local variation (shape change) to be made. Snapshots from such a movie are shown in Figure 4.)

\section{Meandering in the Plane}

We show that Euclidean symmetry accounts for the meandering of spiral waves (in contrast to the nonuniform rigid rotation described in the previous subsection). In addition, we recover results of $[3,42,13]$ on resonance and unbounded linear drift.

Again, the center bundle is a trivial bundle $X=\mathbf{S E}(2) \times \mathbf{C}$ and, as in [13, Lemma 4.1], 
equivariance implies that the equations on the center bundle are given by

$$
\begin{aligned}
\dot{p} & =e^{i \varphi} f(q, \lambda) \\
\dot{\varphi} & =F^{\varphi}(q, \lambda) \\
\dot{q} & =F^{q}(q, \lambda)
\end{aligned}
$$

where $(p, \varphi) \in \mathbf{C} \times S^{1} \cong \mathbf{S E}(2)$ denotes the group variables (translation and rotation) and $q \in \mathbf{C}$ denotes the Hopf or shape variables. We have $f(0,0)=0, F^{\varphi}(0,0)=\omega_{\text {rot }}$ and $\left(d F^{q}\right)_{0,0}=i \omega_{\mathrm{bif}}$.

The solutions of the $\dot{q}$ and $\dot{\varphi}$ equations are the same as in the circularly symmetric situation in the previous subsection. We solve for a branch of $2 \pi / \omega_{\text {bif }}(\lambda)$ periodic solutions $q(t, \lambda)$ and obtain

$$
\varphi(t, \lambda)=\omega_{\text {rot }}(\lambda) t+\sqrt{\lambda} \psi(t, \lambda)
$$

where $\psi(t, \lambda)$ is $2 \pi / \omega_{\mathrm{bif}}(\lambda)$ periodic.

The $\dot{p}$ equation becomes

$$
\dot{p}=\sqrt{\lambda} e^{i \omega_{\mathrm{rot}}(\lambda) t} g(t, \lambda)
$$

where $g(t, \lambda)$ is $2 \pi / \omega_{\text {bif }}(\lambda)$ periodic.

\section{Nonresonance versus Resonance}

To determine the form of $p(t, \lambda)$, we write $g$ as a Fourier series

$$
g(t, \lambda)=\sum_{j \in \mathbf{Z}} g_{j}(\lambda) e^{i j \omega_{\mathrm{bif}}(\lambda) t} .
$$

Following [13] we integrate (3.3) term by term noting that the result depends on whether the center bundle equations are nonresonant or resonant.

Recall that the center bundle equations are nonresonant if for every integer $n$

$$
\omega_{\text {rot }}(\lambda)+n \omega_{\text {bif }}(\lambda) \neq 0 .
$$

Assuming nonresonance, compute that

$$
p(t, \lambda)=\sqrt{\lambda} \sum_{j \in Z} \frac{1}{i\left(\omega_{\mathrm{rot}}(\lambda)+j \omega_{\mathrm{bif}}(\lambda)\right)} g_{j}(\lambda) e^{i\left(\omega_{\mathrm{rot}}(\lambda)+j \omega_{\mathrm{bif}}(\lambda)\right) t},
$$

which is quasiperiodic. 
Now suppose that for a specific value of $\lambda$, there is an $n^{\text {th }}$ order resonance

$$
\omega_{\text {rot }}(\lambda)+n \omega_{\text {bif }}(\lambda)=0
$$

for some $n \in \mathbf{Z}$. This time we compute that

$$
p(t, \lambda)=\sqrt{\lambda}\left(\sum_{j \neq n} \frac{1}{i\left(\omega_{\mathrm{rot}}(\lambda)+j \omega_{\mathrm{bif}}(\lambda)\right)} g_{j}(\lambda) e^{i\left(\omega_{\mathrm{rot}}(\lambda)+j \omega_{\mathrm{bif}}(\lambda)\right) t}+g_{n}(\lambda) t\right) .
$$

As shown in Fiedler and Turaev [12], the $\sqrt{\lambda} g_{n}(\lambda)$ term is of order $|n| / 2$ in $\lambda$. We rederive this result as a special case of Proposition 5.4.

To summarize: at nonresonance we have

$$
p(t, \lambda)=\sqrt{\lambda} p_{0}(t, \lambda)
$$

which is quasiperiodic typically with two independent frequencies, and at $n^{\text {th }}$ order resonance, we have

$$
p(t, \lambda)=\sqrt{\lambda} p_{0}(t, \lambda)+\lambda^{|n| / 2} p_{1}(\lambda) t,
$$

where $p_{0}(t, \lambda)$ is periodic. Of course, at resonance the frequencies $\omega_{\text {rot }}(\lambda)$ and $\omega_{\text {bif }}(\lambda)$ are rationally related so that $p_{0}$ is periodic instead of quasiperiodic.

\section{Implications of (3.4) and (3.5) for Patterns in Physical Space}

Away from resonance, the quasiperiodic fluctuation of the translation $p(t, \lambda)$ coupled with the nonuniform rotation $\varphi(t, \lambda)$ leads to quasiperiodic meander in the plane. In particular, as we showed in Figure 6, the translation $p(t, \lambda)$ forces petal type motion which is the defining characteristic of meander. This motion in Figure 6(c) (and Figure 5) should be contrasted with the nonuniform motion of Figure 6(b) (and Figure 4) where translation symmetry is not present.

Near resonance, we now have linear drift (the term $\lambda^{|n| / 2} p_{1}(\lambda) t$ ) superimposed on the quasiperiodic meander. The effect of $n^{\text {th }}$ order resonance is present for all values of $n$, but the time that it takes for the linear drift to be discernible grows with $n$.

\section{Modulated Waves in Circular Domains — Flames}

In this section, we consider Hopf bifurcation from rotating waves with possibly nontrivial isotropy $\Sigma_{\text {rot }}=\mathbf{Z}_{k}, k \geq 1$, in systems with symmetry group $\Gamma=\mathbf{O}(2)($ or $\Gamma=\mathbf{S O}(2)$ ). 
Rand [35] classified the various modulated rotating waves that occur in terms of their spatial and spatiotemporal symmetries. We begin by rederiving Rand's classification. Then, we proceed as in the case $k=1$ to obtain further information about the behavior of these solutions in physical space beyond their symmetry properties.

\section{Classification of Modulated Rotating Waves}

Recall that Hopf bifurcation from a rotating wave corresponds to Hopf bifurcation from an equilibrium for the $\Sigma_{\text {rot }}$ equivariant normal vector field $g: N_{0} \rightarrow N_{0}$. Let $z \in \mathbf{C}$ denote coordinates for the critical eigenspace in $N_{0}$. The action of the isotropy subgroup $\Sigma_{\text {rot }}=Z_{k}$ on $z \in \mathbf{C}$ is generated by

$$
R_{\frac{2 \pi}{k}} \cdot z=e^{2 \pi i m / k} z,
$$

for some $m=0,1, \ldots,[k / 2]$. Rand's classification of modulated rotating waves is essentially given in terms of the integers $k$ and $m$. One difficulty is that the integer $m$ has no direct physical interpretation. Following Rand (though with different notation) we introduce the derived integers $d \geq 1$ and $\alpha \in\left\{0,1, \ldots, \frac{k}{d}-1\right\}$ where

$$
d=\operatorname{gcd}(k, m), \quad \alpha m \equiv d \bmod k .
$$

We show that the integers $k, d$ and $\alpha$ are quantities that can be determined experimentally. (The integers $(k, d, \alpha)$ correspond to Rand's integers $(m, s, n)$.)

Remark 4.1 We note that $\alpha$ is the multiplicative inverse of $m / d$ modulo $k / d$. Hence, given $k, d$ and $\alpha \geq 1$ we can recover $m$ through the equation $\alpha(m / d) \equiv 1 \bmod (k / d)$. (When $\alpha=0$, we have $k=d$ and $m=0$.)

The isotropy subgroup $\Sigma_{\text {bif }}$ of the bifurcating modulated rotating wave is given by the kernel of the action (4.1) on the critical eigenspace. Hence, $\Sigma_{\text {bif }}=\mathbf{Z}_{d}$ where $d=\operatorname{gcd}(k, m)$. Thus, the integers $k$ and $d$ correspond to the spatial symmetry (at a fixed moment in time) of the rotating wave $\left(\Sigma_{\text {rot }}=\mathbf{Z}_{k}\right)$ and the modulated rotating wave $\left(\Sigma_{\text {bif }}=\mathbf{Z}_{d}\right)$.

Next, we show that the integer $\alpha$ determines the spatiotemporal symmetry of the modulated rotating wave. Let $y(t, \lambda)$ be the solution to the (infinite-dimensional) normal vector field equations on $N_{0}$. Then $y$ is $T(\lambda)$ periodic where $T(\lambda)=2 \pi / \omega_{\text {bif }}(\lambda)$. In addition, there is the nontrivial spatiotemporal symmetry [14]

$$
y\left(t+\frac{m}{k} T(\lambda), \lambda\right)=R_{\frac{2 \pi}{k}} \cdot y(t, \lambda),
$$

We now compute the minimal spatiotemporal symmetry corresponding to these two symmetries. (We say that $y(t+T)=\gamma y(t)$ is a minimal spatiotemporal symmetry if $T>0$ and $\gamma \in \Sigma_{\text {rot }}$ such that $T$ is minimal.) 
Proposition 4.2 The normal vector field solution $y$ has the minimal spatiotemporal symmetry

$$
y\left(t+\frac{d}{k} T(\lambda), \lambda\right)=\left(R_{\frac{2 \pi}{k}}\right)^{\alpha} \cdot y(t, \lambda) .
$$

Proof Since $d=\operatorname{gcd}(k, m)$, we have $\alpha m+\beta k=d$ for some $\beta \in \mathbf{Z}$. Hence,

$$
\frac{d}{k} T(\lambda)=\alpha \frac{m}{k} T(\lambda)+\beta T(\lambda)
$$

It follows from $T(\lambda)$ periodicity and (4.2) that

$$
y\left(t+\frac{d}{k} T(\lambda), \lambda\right)=y\left(t+\alpha \frac{m}{k} T(\lambda), \lambda\right)=\left(R_{2 \pi / k}\right)^{\alpha} \cdot y(t, \lambda),
$$

verifying that (4.3) is a spatiotemporal symmetry of $y$.

Next we show that (4.3) is the minimal spatiotemporal symmetry. Recall that in Hopf bifurcation, $T(\lambda)$ is the minimal period of $y(t, \lambda)$. From now on, we suppress the $\lambda$ 's. Suppose that $y(t+S)=\gamma y(t)$ for some $S>0, \gamma \in \Sigma_{\text {rot }}$, so

$$
y(t+S)=\left(R_{2 \pi / k}\right)^{j} \cdot y(t)
$$

where $j \geq 1$. We must show that $S \geq \frac{d}{k} T$. Now,

$$
y\left(t+\frac{k}{d} S\right)=\left(R_{2 \pi / d}\right)^{j} \cdot y(t)=y(t)
$$

since $\Sigma_{\text {bif }}=\mathbf{Z}_{d}$. Hence $\frac{k}{d} S$ is a multiple of $T$. Since $S>0$, we have $S \geq \frac{d}{k} T$ as required.

By the results of Krupa [27], the symmetry (4.3) corresponds to an exact spatiotemporal symmetry of the full modulated rotating wave solution $u(t, \lambda)$ modulo the drift along the SO(2)-group orbit. Thus, in a suitable rotating frame, the modulated rotating wave reduces to a periodic solution and the integer $\alpha$ in Proposition 4.2 determines the spatiotemporal symmetry of that periodic solution.

\section{Implications of the Classification of Modulated Rotating Waves for Flames}

As pointed out in [7], Rand's classification of modulated rotating waves applies directly to Gorman's flame experiment. Indeed, the "hopping modes" or "ponies on a merry-go-round" that are observed in the physical and numerical experiments seem to have the behavior expected of modulated rotating waves. 
In the flame experiments, Gorman et al. [19] observe modulated rotating waves in the form of cellular states with $k$ not quite identical cells. (Presumably, these states bifurcate from a rotating wave with $k$ identical cells; this transition has been observed in numerical simulations [7] but not yet in the experiments.) The entire state is rotating but there are additional 'events' where successive cells fire one after the other in identical fashion. In terms of the classification, these modulated rotating waves bifurcate from a state with $k>1$ identical cells to a state with no identical cells $d=1$ and a spatiotemporal symmetry corresponding to $\alpha=1$. States with $d=k$ identical cells before and after bifurcation (the nonsymmetry breaking case) are also observed.

The hopping modes of Gorman et al. [17] appear to be modulated rotating waves as above, but with additional structure that is beyond the scope of this paper. See Palacios et al. [32] for results concerning the additional structure of the hopping modes.

In numerical simulations, Bayliss et al. [7] obtain a number of modulated rotating waves arising through symmetry breaking $(m>0$ or equivalently $d<k)$ bifurcations from rotating waves. They particularly emphasize bifurcations from rotating waves with four identical cells $(k=4)$ and with seven identical cells $(k=7)$.

According to the classification, there are two distinct possibilities corresponding to $k=4$ : the cases $m=1$ and $m=2$. The case $m=1$ is shown in [7, Figures 3 and 4] and corresponds to the data $d=1, \ell=4$ and $\alpha=1$. Note that all of the symmetry is broken $(d=1)$ and that after passing to the rotating frame, time-shift by a quarter of the period $(\ell=4)$ is the same as a quarter rotation $(\alpha / k=1 / 4)$. The case $m=2$ is the "half period modulated rotating wave" shown in [7, Figure 8] and corresponds to the data $d=2, \ell=2$, and $\alpha=1$. This time, part of the symmetry is preserved $(d=2)$ and in the rotating frame, time-shift by half a period $(\ell=2)$ is the same as a quarter rotation $(\alpha / k=1 / 4)$. The modulated rotating wave in [7, Figure 3] ("Pushmi-Pullyu") appears to have more structure than the "breathing" modulated rotating wave in [7, Figure 4]. Again, this additional structure lies outside the scope of this paper.

The classification yields three possibilities corresponding to $k=7: m=1, m=2$ and $m=3$. In each of these cases, $d=1, k=\ell$, and all the symmetry is broken. The case $m=1$ yields $\alpha=1$ as is shown in [7, Figure 11]. The case $m=2$ yields $\alpha=4$ as is shown in [7, Figure 10]. Finally, the case $m=3$ yields $\alpha=5$ as is shown in [7, Figure 9] (though we note that the numbering is such that this case appears to correspond more closely to the mathematically identical case $m=4$ and $\alpha=2$ ). The last two cases have $\alpha>1$, leading to the terminology "jumping ponies on a merry-go-round". 


\section{The Center Bundle Equations}

The analysis of the "nonuniformly rotating" nature of the modulated rotating waves bifurcating from rotating waves with isotropy $\mathbf{Z}_{k}$ is similar to the analysis in Section 3 of the case $k=1$. However, to solve the equations on the center bundle, it is necessary to pass to the skew product construction of [11]. These equations have the same form as the center bundle equations for $k=1$ except that there are the $\Sigma_{\text {rot }}$ equivariance conditions

$$
\begin{aligned}
F^{\varphi}(\sigma q, \lambda) & =\sigma F^{\varphi}(q, \lambda) \sigma^{-1}, \\
F^{q}(\sigma q, \lambda) & =\sigma F^{q}(q, \lambda),
\end{aligned}
$$

for $\sigma \in \Sigma_{\text {rot }}$. The right-hand-side of (4.4) reduces to $F^{\varphi}(q, \lambda)$ since $\mathbf{S O}(2)$ is abelian, so that the condition simply states that $F^{\varphi}$ is $\Sigma_{\text {rot }}$ invariant:

$$
F^{\varphi}(\sigma q, \lambda)=F^{\varphi}(q, \lambda) .
$$

In (4.5) and (4.6), the action of $\sigma \in \Sigma_{\text {rot }}$ on $q$ (and $F^{q}$ ) corresponds to the action (4.1) on the critical eigenfunctions, namely

$$
R_{\frac{2 \pi}{k}} \cdot q=e^{2 \pi i m / k} q
$$

When $m=0$ (so $\Sigma_{\text {bif }}=\Sigma_{\text {rot }}=\mathbf{Z}_{k}$ ), there is no restriction on $F^{\varphi}$ or $F^{q}$ and the results are the same as when $k=1$.

In the remaining cases $(m \geq 1), \Sigma_{\text {bif }}$ is a proper subgroup of $\Sigma_{\text {rot }} ; \Sigma_{\text {bif }}=\mathbf{Z}_{d}$ where $d=\operatorname{gcd}(k, m)$. Set $\ell=k / d$ and $T(\lambda)=2 \pi / \omega_{\text {bif }}(\lambda)$.

Lemma 4.3 Generically,

$$
F^{\varphi}(q(t, \lambda), \lambda)=\omega_{\text {rot }}(\lambda)+\lambda^{\ell / 2} \psi_{0}(t, \lambda)
$$

where $\psi_{0}(t, \lambda)$ has minimal period $T(\lambda) / \ell$.

Proof By Proposition 4.2, the solution $q(t, \lambda)$ to the $\dot{q}$ equation has the minimal spatiotemporal symmetry

$$
q\left(t+\frac{1}{\ell} T(\lambda), \lambda\right)=\left(R_{\frac{2 \pi}{k}}\right)^{\alpha} \cdot q(t, \lambda)=e^{2 \pi i \alpha m / k} q(t, \lambda)=e^{2 \pi i / \ell} q(t, \lambda) .
$$

This spatiotemporal symmetry combined with the invariance condition (4.6) implies that $F^{\varphi}(q(t, \lambda), \lambda)$ has period $T(\lambda) / \ell$. Since $F^{\varphi}$ is an arbitrary invariant function, generically this period is minimal. Verification of the exponent $\ell / 2$ of $\lambda$ is postponed until Section 8 .

It follows from the lemma that the rigid rotation along the group orbit is given by

$$
\varphi(t, \lambda)=\omega_{\text {rot }}(\lambda) t+\lambda^{\ell / 2} \psi(t, \lambda)
$$

where $\psi(t, \lambda)$ has minimal period $T(\lambda) / \ell$. 


\section{Implications of (4.8) for Patterns in Physical Space}

From the point of view of this paper, the nonuniformly rotating states and the ponies on a merry-go-round observed in the flame experiments $[19,33]$ and in numerical simulations [7] can be studied together as modulated rotating waves bifurcating from a rotating wave. Near onset, all of these states are nonuniformly rotating waves, the only distinction being the rate $\ell / 2$ at which the nonuniformity in the rotation speed scales with the bifurcation parameter. The nonuniformity is most visible when $k=d(\ell=1)$. This corresponds to the nonsymmetry breaking case $\Sigma_{\text {bif }}=\Sigma_{\text {rot }}$.

In the symmetry breaking case $\Sigma_{\text {bif }} \neq \Sigma_{\text {rot }}(\ell=k / d>1)$, the nonuniformity of the rotation speed is less apparent (though careful experiments should still pick up this feature). In addition, further away from the bifurcation point, the spatiotemporal symmetry becomes dominant and leads to ponies on a merry-go-round. The scenario we envisage for the flame experiment is that a uniformly rotating cellular state with $k$ identical cells bifurcates to a nonuniformly rotating state with $k$ cells that are not all identical and then gradually (as the bifurcation parameter is varied) transforms into ponies on a merry-go-round. It should be noted that this second transition is not a dynamical bifurcation - neither the symmetry nor the qualitative dynamics is altered. Rather, we have an exchange in dominance of the drift variables (which drive the nonuniform rotation) and the shape variables (which drive the spatiotemporal symmetry).

\section{Reversing States}

The nonuniformly rotating patterns that arise in the flame experiments should be contrasted with the "direction-reversing traveling waves" analyzed in Landsberg and Knobloch [28]. The setting in [28] is Hopf bifurcation from a circle of equilibria with reflection symmetry, so $\Gamma=\mathbf{O}(2)$ and $\Sigma_{\text {rot }}=\mathbf{Z}_{2}$ where $\mathbf{Z}_{2}$ is generated by a reflection. Hopf bifurcation with $\mathbf{Z}_{2}$ symmetry [15] leads to branches of periodic solutions with no spatial symmetry but where the reflection symmetry reappears as a spatiotemporal symmetry (with a half period phase shift). Such solutions do not drift [27] and so we have periodic solutions (instead of modulated rotating waves) in the full $\mathbf{O}(2)$-equivariant problem. In particular, there is zero mean drift.

In the notation used in this paper, the results of [28] say that the rigid rotation variable $\varphi$ has the form

$$
\varphi(t)=\lambda^{1 / 2} \psi(t, \lambda),
$$

where $\psi(t, \lambda)$ has period $2 \pi / \omega_{\text {bif }}(\lambda)$. In particular, $\omega_{\text {rot }}(\lambda) \equiv 0$. Ignoring shape changes, the reversing states are observed to rigidly rotate (or travel) in one direction and then in the other direction, reversing direction periodically. 


\section{Ratcheting States}

In the flame experiments, "ratcheting states" are observed [18] which have certain features in common with ponies on a merry-go-round (or the hopping mode). In particular, all of these states are quasiperiodic and reduce to small amplitude periodic states in a rotating frame. In the ratcheting state, however, the magnitude of the nonuniformity in rotation speed is large compared to the the speed of the underlying rotating frame; indeed contragrade motions are observed in ratcheting states. (Unlike the reversing states of [28], there is a nonzero but slow mean drift.)

The following scenario may explain the similarities and differences between ratcheting states and ponies on a merry-go-round. Whereas ponies on a merry-go-round bifurcate from a rotating wave, we propose that the ratcheting states bifurcate from a stationary solution.

Specifically, we propose that ratcheting states arise through a symmetry breaking Hopf bifurcation from a $\mathbf{D}_{k}$ invariant steady state. Applying the results of Krupa [27], we first consider $\mathbf{D}_{k}$ equivariant Hopf bifurcation and then allow for drifts along the $\mathbf{S O}(2)$-group orbit.

Symmetry breaking Hopf bifurcation with $\mathbf{D}_{k}$ symmetry is studied in [15, Chapter XVIII]. We define the integers $m, d, \ell$ just as for the $\mathbf{Z}_{k}$ bifurcations considered so far. Provided that $\ell \geq 3$, there is a four dimensional critical eigenspace and three maximal isotropy subgroups each with two dimensional fixed point subspace. The equivariant Hopf bifurcation yields branches of periodic solutions for each of these isotropy subgroups.

The possibility of drifts along the $\mathbf{S O}(2)$ group orbit means that some of these branches yield quasiperiodic solutions. Two of the branches consist of periodic solutions with either spatial or spatiotemporal reflection symmetry; continuous drifts are then excluded and these are ordinary periodic solutions. The third branch consists of solutions with rotation spatiotemporal symmetry only $\left(\widetilde{\mathbf{Z}}_{\ell}\right.$ in the notation used in [15]). Hence, generically there is drift along the $\mathbf{S O}(2)$-orbit and we have a two frequency modulated rotating wave.

The skew product equations for the modulated rotating wave have a form similar to before, but $\Sigma_{\text {rot }}$ is replaced by $\mathbf{D}_{k}$. In particular, $F^{\varphi}$ satisfies a $\mathbf{D}_{k}$ equivariance condition of the form (4.4), so that $F^{\varphi}(\sigma q, \lambda)=F^{\varphi}(q, \lambda)$ when $\sigma \in \mathbf{D}_{k}$ is a rotation, and $F^{\varphi}(\sigma q, \lambda)=$ $-F^{\varphi}(q, \lambda)$ when $\sigma \in \mathbf{D}_{k}$ is a reflection.

Suppose that $q(t, \lambda)$ is the $\widetilde{\mathbf{Z}}_{\ell}$ branch of periodic solutions in $\mathbf{D}_{k}$ Hopf bifurcation.

Lemma 4.4 Generically,

$$
\int_{0}^{T(\lambda)} F^{\varphi}(q(t, \lambda), \lambda) d t \sim \lambda^{\ell-1}
$$

and

$$
F^{\varphi}(q(t, \lambda), \lambda)=\lambda^{\ell-1} a(\lambda)+\lambda^{\ell / 2} \psi_{0}(t, \lambda),
$$


where $\psi_{0}(t, \lambda)$ has minimal period $T(\lambda) / \ell$.

Proof Verification of the periodicity of $\psi(t, \lambda)$ proceeds as in Lemma 4.3. The exponents $\ell-1$ and $\ell / 2$ of $\lambda$ are verified in Section 8 .

It follows from the lemma that the rigid rotation along the group orbit is given by

$$
\varphi(t, \lambda)=\lambda^{\ell-1} a(\lambda) t+\lambda^{\ell / 2} \psi(t, \lambda),
$$

where $\psi(t, \lambda)$ has minimal period $T(\lambda) / \ell$. (Of course $\omega_{\text {rot }}=0$ in this bifurcation.) Observe that the leading nonconstant term dominates the constant term, since $\ell \geq 3$, leading to contragrade motions as seen in the experiments.

\section{Modulated Rotating Waves in the Plane - Spirals}

We consider the case of Hopf bifurcation from $k$-armed spirals, $k \geq 1$ following [13]. The classification of modulated rotating wave spiral states is identical to that described in Section 4 and we concentrate on the manifestation of the quasiperiodic dynamics as motions in physical space. As pointed out in [13], both meandering and nonuniform rigid rotation is possible in bifurcation from $k$-armed spirals, depending on whether or not all of the symmetry is broken in the bifurcation.

Remark 5.1 In [13], we derived also the conditions for resonance for $k$-armed spirals. Unfortunately, the conditions for resonance when $k \geq 2$, stated in [13, Theorem 5.2], are incorrect. We are grateful to Claudia Wulff for pointing this out to us.

The source of the errors in [13] is as follows. We introduced physical frequencies $\omega_{1}, \omega_{2}$ (corresponding to $\omega_{\text {rot }}$ and $\omega_{\text {bif }}$ in this paper) and frequencies $\widetilde{\omega}_{1}, \widetilde{\omega}_{2}$ corresponding to the skew product equations. The complicated relations between these frequencies in [13] are wrong and in fact $\widetilde{\omega}_{1}=\omega_{1}, \widetilde{\omega}_{2}=\omega_{2}$ (as can be seen for example in the construction of [11]). Indeed, the correct conditions for resonance follow immediately from [13, Theorem 5.5] and are given in (5.5) below.

Passing directly to the skew-product equations [11], we consider the system of ODEs

$$
\begin{aligned}
\dot{p} & =e^{i \varphi} f(q, \lambda) \\
\dot{\varphi} & =F^{\varphi}(q, \lambda) \\
\dot{q} & =F^{q}(q, \lambda)
\end{aligned}
$$

where $(p, \varphi) \in \mathbf{C} \times S^{1} \cong \mathbf{S E}(2)$ denotes the group variables (translation and rotation) and $q \in \mathbf{C}$ denotes the Hopf or shape variables. 
Just as in Section 4, we introduce the integers $m, d$ and $\ell$. The action of the isotropy subgroup $\Sigma_{\text {rot }}=\mathbf{Z}_{k}$ is generated by $q \mapsto e^{2 \pi i m / k} q$ where $m=0,1, \ldots,[k / 2]$. We set $d=$ $\operatorname{gcd}(k, m)$ and $\ell=k / d$. Define $\omega_{\text {rot }}(\lambda), \omega_{\text {bif }}(\lambda)$ as before and set $T(\lambda)=2 \pi / \omega_{\text {bif }}(\lambda)$.

The skew-product equations are subject to the $\Sigma_{\text {rot }}$ equivariance conditions

$$
\begin{aligned}
f(\sigma q, \lambda) & =\sigma f(q, \lambda), \\
F^{\varphi}(\sigma q, \lambda) & =F^{\varphi}(q, \lambda), \\
F^{q}(\sigma q, \lambda) & =\sigma F^{q}(q, \lambda),
\end{aligned}
$$

for $\sigma \in \Sigma_{\text {rot }}$. Again, the action of $\sigma$ on $q$ and $F^{q}$ is defined by the integer $m$, whereas the action of $\sigma$ on the right-hand-side of (5.1) is group multiplication.

The solution of the $\dot{q}$ and $\dot{\varphi}$ equations is unchanged. We solve for a branch of $T(\lambda)$ periodic solutions $q(t, \lambda)$ and obtain

$$
\varphi(t, \lambda)=\omega_{\text {rot }}(\lambda) t+\lambda^{\ell / 2} \psi(t, \lambda),
$$

where $\psi(t, \lambda)$ has minimal period $T(\lambda) / \ell$.

\section{The Case when $k$ and $m$ are not Coprime}

Proposition 5.2 When $k$ and $m$ are not coprime, $p(t, \lambda) \equiv 0$.

Proof Condition (5.1) can be written as

$$
f\left(e^{2 \pi i m / k} q, \lambda\right)=e^{2 \pi i / k} f(q, \lambda) .
$$

In particular,

$$
e^{2 \pi i / d} f(q, \lambda)=f\left(e^{2 \pi i m / d} q, \lambda\right)=f(q, \lambda) .
$$

Since $d \geq 2$, it follows that $f \equiv 0$.

\section{The Case when $k$ and $m$ are Coprime}

Since $k$ and $m$ are coprime, $d=1$ and $\ell=k$. As before, we solve for a branch of $T(\lambda)$ periodic solutions $q(t, \lambda)$ and obtain

$$
\varphi(t, \lambda)=\omega_{\text {rot }}(\lambda) t+\lambda^{k / 2} \psi(t, \lambda),
$$

where $\psi(t, \lambda)$ has minimal period $T(\lambda) / k$. The $\dot{p}$ equation becomes

$$
\dot{p}=e^{i \omega_{\mathrm{rot}}(\lambda) t} g(t, \lambda),
$$


where

$$
g(t, \lambda)=e^{i \lambda^{k / 2} \psi(t, \lambda)} f(q(t, \lambda), \lambda)
$$

Proposition 5.3 Suppose that for every integer $j$ satisfying $j m \equiv 1 \bmod k$, we have

$$
\omega_{\text {rot }}(\lambda)+j \omega_{\text {bif }}(\lambda) \neq 0 \text {. }
$$

Let $\beta$ be least positive integer such that $\beta m= \pm 1 \bmod k$. Then

$$
p(t, \lambda)=\lambda^{\beta / 2} p_{0}(t, \lambda)
$$

is quasiperiodic (with typically two independent frequencies).

With the exception of the order of $p$ in $\lambda$ and the difficulty with the nonresonance conditions (5.3) (see Remark 5.1), this proposition was proved in [13]. For ease in exposition we give a complete proof of Proposition 5.3.

Proof Recall from (4.7) that $q(t+T(\lambda) / k, \lambda)=e^{2 \pi i \alpha m / k} q(t, \lambda)$. Using (5.2), we compute that the minimal spatiotemporal symmetry of $f(q(t, \lambda), \lambda)$ is given by

$$
f\left(q\left(t+\frac{1}{k} T(\lambda), \lambda\right), \lambda\right)=e^{2 \pi i \alpha / k} f(q(t, \lambda), \lambda) .
$$

Since $\psi$ is $T(\lambda) / k$ periodic, $g$ also possesses the minimal spatiotemporal symmetry

$$
g\left(t+\frac{1}{k} T(\lambda), \lambda\right)=e^{2 \pi i \alpha / k} g(t, \lambda) .
$$

Next we write $g$ as a Fourier series

$$
g(t, \lambda)=\sum g_{j}(\lambda) e^{i j \omega_{\mathrm{bif}}(\lambda) t}
$$

By (5.4), this series reduces to summation over those integers $j$ satisfying $j m \equiv 1 \bmod k$. Multiplying by $e^{i \omega_{\text {rot }}(\lambda) t}$ and integrating, we obtain a quasiperiodic function $p(t, \lambda)$ if and only if the nonresonance hypotheses (5.3) are satisfied.

The verification of the exponent $\beta / 2$ is postponed until Section 8 
The case when $k$ and $m$ are coprime with resonance

Now suppose that there is an $n^{\text {th }}$ order resonance

$$
\omega_{\text {rot }}+n \omega_{\text {bif }}=0
$$

for some $n \in \mathbf{Z}$ satisfying $n m \equiv 1 \bmod k$.

Proposition 5.4 At an $n^{\text {th }}$ order resonance,

$$
p(t, \lambda)=\lambda^{\beta / 2} p_{0}(t, \lambda)+\lambda^{|n| / 2} p_{1}(\lambda) t
$$

where $p_{0}(t, \lambda)$ is periodic.

Proof By the proof of Proposition 5.3, it remains to verify the exponent $|n| / 2$. This verification is postponed until Section 8.

\section{Implications of Propositions 5.2, 5.3 and 5.4 for Patterns in Physical Space}

When $\Sigma_{\text {bif }} \neq \mathbf{1}$, we have the prediction [13] of rigid nonuniform rotation and no meander. To this, we can add that the nonuniformity in the rotation is most noticeable when $\Sigma_{\text {bif }}=$ $\Sigma_{\text {rot }}=\mathbf{Z}_{k}(k \geq 2)$. When $\Sigma_{\text {bif }} \neq \Sigma_{\text {rot }},\left(\Sigma_{\text {bif }}=\mathbf{Z}_{d}, 1<d<k\right)$, there is the additional spatiotemporal symmetry where the arms of the spiral, which are no longer identical, 'fire' after equal periods of time in identical fashion.

When $\Sigma_{\text {bif }}=\mathbf{1}$, we obtain meander and linear drift as for the case of one-armed spirals. Away from resonance, we predict meandering, with the size of the petals depending on the integer $\beta$. The petals are most well-defined when $\beta=1$. This occurs in the case $k=1, m=0$ (one-armed spirals) and also in the cases where $k$ is arbitrary and $m=1$.

\section{Modulated Wavy Vortices in Taylor-Couette}

As discussed in the introduction, the Taylor-Couette experiment is often modeled as having $\mathbf{S O}(2) \times \mathbf{O}(2)$ symmetry, where $\mathbf{S O}(2)$ consists of azimuthal rotations and $\mathbf{O}(2)$ consists of axial translations and an up-down flip $\kappa$. It turns out that most of our results are unchanged if we just assume $\mathbf{S O}(2) \times \mathbf{Z}_{2}$ symmetry (azimuthal rotations and the up-down flip), but there are subtle differences in the results which we describe at the end of the section.

Recall that the isotropy subgroup of wavy vortices is given by $\Sigma_{\text {rot }}=\mathbf{Z}_{2 k}$ consisting of pure azimuthal rotations $\mathbf{Z}_{k} \subset \mathbf{S O}(2)$ (generated by $(2 \pi / k, 0) \in \mathbf{S O}(2) \times \mathbf{S O}(2)$ together with symmetries in $\mathbf{Z}_{2 k} \sim \mathbf{Z}_{k}$ that are azimuthal rotations combined with the up-down flip). Hence $\Sigma_{\text {rot }}$ is generated by a single glide reflection: rotation by $(\pi / k, 0)$ followed by the flip $\kappa$. 


\section{Extended Classification of Modulated Rotating Waves in Taylor-Couette}

The representation of the isotropy subgroup $\Sigma_{\mathrm{rot}}=\mathbf{Z}_{2 k}$ is generated by $q \mapsto e^{\pi i m / k} q$ for some $m=0,1, \ldots, k$. As in Section 4 , we obtain a classification of the possible types of modulated rotating waves in terms of the integers $(2 k, d, \alpha)$ where $d=\operatorname{gcd}(2 k, m)$ and $\alpha m \equiv d \bmod 2 k$. Again, $k$ and $d$ denote the instantaneous symmetry of wavy vortices and modulated wavy vortices, while $\alpha$ measures the spatiotemporal symmetry.

We distinguish between modulated wavy vortices which break all the spatial glide reflection symmetry of the wavy vortices and those that retain some of this symmetry. In the terminology of [9], Gorman-Swinney or GS flows are modulated wavy vortices that break the glide reflection symmetry while Zhang-Swinney or ZS flows retain some of the glide reflection symmetry. Note that GS flows occur when $2 k / d$ is even and ZS flows occur when $2 k / d$ is odd. To see this, let $\rho$ be a generator of $\Sigma_{\text {rot }}=\mathbf{Z}_{2 k}$ and observe that $\rho^{j}$ is a glide reflection if and only if $j$ is odd. But $\Sigma_{\text {bif }}=\mathbf{Z}_{d}$ is generated by $\rho^{2 k / d}$ and hence contains odd powers of $\rho$ precisely when $2 k / d$ is odd.

Both kinds of modulated wavy vortices have been observed in experiments. The GS flows are the original modulated wavy vortices of Gorman and Swinney [20, 21] and do not possess spatial glide reflection symmetry (though such symmetries necessarily appear as spatiotemporal symmetries). The ZS flows were obtained more recently in experiments of Zhang and Swinney [43] and possess spatial glide reflection symmetry, as noted in [9].

In Section 4, we described Rand's classification [35] of modulated rotating waves in systems with $\mathbf{S O}(2)$ symmetry. This classification was particularly geared towards modulated wavy vortices for which the symmetry group is actually $\mathbf{S O}(2) \times \mathbf{O}(2)$ (or at least $\mathbf{S O}(2) \times \mathbf{Z}_{2}$ which leads to the same results). In particular, the isotropy subgroup of wavy vortices is given by $\Sigma_{\text {rot }}=\mathbf{Z}_{2 k}$ and does not lie in $\mathbf{S O}(2)$, whereas Rand has $\Sigma_{\text {rot }}=\mathbf{Z}_{k} \subset \mathbf{S O}(2)$. In particular, we obtain twice as many states as does [35]: for each solution in [35], there corresponds a GS flow and a ZS flow.

\section{The Center Bundle Equations}

The skew-product equations have the form

$$
\begin{aligned}
\dot{\varphi_{1}} & =F^{\varphi_{1}}(q, \lambda) \\
\dot{\varphi_{2}} & =F^{\varphi_{2}}(q, \lambda) \\
\dot{q} & =F^{q}(q, \lambda),
\end{aligned}
$$


where $\varphi_{1}, \varphi_{2}$ and $q$ denote the azimuthal, axial and shape variables. In addition, $F^{\varphi_{1}}(0,0)=$ $\omega_{\text {rot }}, F^{\varphi_{2}}(0,0)=0$ and $\left(d F^{q}\right)_{0,0}=i \omega_{\text {bif }} q$. We have the $\Sigma_{\text {rot }}$ equivariance conditions

$$
\begin{gathered}
F^{\varphi_{1}}\left(e^{\pi i m / k} q, \lambda\right)=F^{\varphi_{1}}(q, \lambda), \\
F^{\varphi_{2}}\left(e^{\pi i m / k} q, \lambda\right)=-F^{\varphi_{2}}(q, \lambda) .
\end{gathered}
$$

Proposition 6.1 Let $d=\operatorname{gcd}(2 k, m), \ell=2 k / d, T(\lambda)=2 \pi / \omega_{\text {bif }}(\lambda)$ and set $\omega_{\text {rot }}(\lambda)=\frac{1}{T(\lambda)} \int_{0}^{T(\lambda)} F^{\varphi_{1}}(q(t, \lambda), \lambda) d t$. Generically, the azimuthal variation is given by

$$
\varphi_{1}(t, \lambda)=\omega_{\text {rot }}(\lambda) t+\lambda^{\ell / 2} \psi_{1}(t, \lambda)
$$

where $\psi_{1}(t, \lambda)$ has minimal period $T(\lambda) / \ell$.

When $\ell$ is even, generically the axial variation is given by

$$
\varphi_{2}(t, \lambda)=\lambda^{\ell / 4} \psi_{2}(t, \lambda)
$$

where $\psi_{2}(t, \lambda)$ has minimal period $2 T(\lambda) / \ell$. When $\ell$ is odd, $\varphi_{2} \equiv 0$.

Proof The vector field $F^{\varphi_{1}}$ satisfies the same invariance condition as the vector field $F^{\varphi}$ in Section 4 (with $k$ replaced by $2 k$ ). Hence, the expression for $\varphi_{1}(t, \lambda)$ is immediate from Lemma 4.3.

Suppose that $\ell$ is odd. We compute that

$$
\left(e^{\pi i m / k}\right)^{\ell}=\left(e^{2 \pi i m / d \ell}\right)^{\ell}=e^{2 \pi i m / d}=1 .
$$

It follows from the equivariance condition satisfied by $F^{\varphi_{2}}$ that

$$
F^{\varphi_{2}}(q, \lambda)=F^{\varphi_{2}}\left(\left(e^{\pi i m / k}\right)^{\ell} q, \lambda\right)=(-1)^{\ell} F^{\varphi_{2}}(q, \lambda)=-F^{\varphi_{2}}(q, \lambda) .
$$

Hence $F^{\varphi_{2}} \equiv 0$.

Next suppose that $\ell$ is even. By definition, $\alpha m \equiv d \bmod 2 k$ from which it follows that $\alpha(m / d) \equiv 1 \bmod \ell$. Hence $\alpha$ and $m / d$ are odd. As in equation $(4.7)$, we have $q(t+$ $T(\lambda) / \ell, \lambda)=\left(e^{\pi i m / k}\right)^{\alpha} q(t, \lambda)$. Therefore,

$F^{\varphi_{2}}(q(t+T(\lambda) / \ell, \lambda), \lambda)=F^{\varphi_{2}}\left(\left(e^{\pi i m / k}\right)^{\alpha} q(t, \lambda), \lambda\right)=(-1)^{\alpha} F^{\varphi_{2}}(q(t, \lambda), \lambda)=-F^{\varphi_{2}}(q(t, \lambda), \lambda)$.

It follows that $F^{\varphi_{2}}(q(t, \lambda), \lambda)$ is $2 T(\lambda) / \ell$ periodic. Again, this period is generically minimal. The computation of the exponent $\ell / 4$ is similar to previous calculations. 


\section{Implications of Proposition 6.1 for Patterns in Physical Space}

All bifurcations lead to doubly quasiperiodic motion that includes nonuniform azimuthal rotation, and none lead to axial drift. The GS flows, but not the ZS flows, possess an additional axial oscillation, analogous to the reversing states of [28].

The case $\ell=2 k / d$ odd corresponds to the ZS flows (spatial glide reflection symmetry). We predict, near the bifurcation point, that the dynamics of the modulated rotating wave in physical space consists of approximately rigid rotation about the axis of the cylinder with nonuniform speed just as in Section 4. Again, we expect that there is a further transition in which the spatiotemporal symmetry gradually dominates the nonuniform rotation.

The case $\ell=2 k / d$ even corresponds to the GS flows (no spatial glide reflection symmetry). Apart from the obvious changes in the spatial and spatiotemporal symmetry, we expect that near the bifurcation point there is a rigid axial oscillation in addition to the nonuniform rotation. (We note that there are still only two independent frequencies.)

Finally, we remark that the distinction between the GS and ZS flows may provide a means for testing between the modeling assumptions of $\mathbf{S O}(2) \times \mathbf{O}(2)$ and $\mathbf{S O}(2) \times \mathbf{Z}_{2}$ symmetry. With the first symmetry group, we expect axial oscillations for the GS flows but not for the ZS flows. With the second symmetry group, we expect no axial oscillations for either state, since there is no longer an axial translation variable. By Proposition 6.1, any axial oscillation of the GS flows would be most apparent when $d=k$ (that is, only the glide reflection symmetry is broken).

\section{Numerical Verification}

In this section, we describe how we obtained the graphical representation of our results in Figures 4, 5, 6 and 7 . We work throughout with the specific center bundle equations

$$
\begin{aligned}
\dot{p} & =e^{i \varphi} q \\
\dot{\varphi} & =\omega_{\text {rot }}+\operatorname{Re} q \\
\dot{q} & =\left(\lambda+i \omega_{\text {bif }}\right) q-|q|^{2} q .
\end{aligned}
$$

These are a special case of the center bundle equations for the problems with $\mathbf{E}(2)$ symmetry in Section 5. Removing the $\dot{p}$ equation we include the problems with $\mathbf{O}(2)$ symmetry in Section 4. 
The solution to the center bundle equations is given by

$$
\begin{aligned}
p(t) & =\frac{\sqrt{\lambda}}{i\left(\omega_{\mathrm{rot}}+\omega_{\mathrm{bif}}\right)} e^{i\left(\omega_{\mathrm{rot}}+\omega_{\mathrm{bif}}\right) t}+O\left(\lambda^{3 / 2}\right) \\
\varphi(t) & =\omega_{\mathrm{rot}} t+\frac{\sqrt{\lambda}}{\omega_{\mathrm{bif}}} \sin \omega_{\mathrm{bif}} t \\
q(t) & =\sqrt{\lambda} e^{i \omega_{\mathrm{bif}} t}
\end{aligned}
$$

Provided that there are no high order resonances (no resonances with $|n| \geq 2$ ) it seems reasonable to discard the $O\left(\lambda^{3 / 2}\right)$ terms in $p(t)$.

Next we describe how to bring in the planar spatial dependence. We suppose that the underlying rotating wave (or its observable) is given by a pure Fourier-Bessel mode

$$
v_{0}(r, \theta)=\operatorname{Im}\left(J_{1}(\mu r) e^{i \theta}\right),
$$

where $J_{1}$ is the first-order Bessel function, and $\mu \in \mathbf{C}$. Such functions have contours which look like one-armed spirals [10]. The time evolution of the rotating wave is given by

$$
v(t)=R_{t} v_{0}=\operatorname{Im}\left(J_{1}(\mu r) e^{i\left(\theta-\omega_{\mathrm{rot}} t\right)}\right),
$$

(taking $R_{t}=\varphi(t)$ with $\lambda=0$ ). We define the 'spiral tip' of the function $v(t): \mathbf{R}^{2} \rightarrow \mathbf{R}$ as the intersection of the contours

$$
\operatorname{Re}\left(J_{1}(\mu r) e^{i\left(\theta-\omega_{\mathrm{rot}} t\right)}\right)=C_{1}, \quad \operatorname{Im}\left(J_{1}(\mu r) e^{i\left(\theta-\omega_{\mathrm{rot}} t\right)}\right)=C_{2},
$$

where $C_{1}, C_{2}$ are constants. The time evolution of the spiral tip of the rotating wave is shown in Figure $6(\mathrm{a})$ with $\omega_{\text {rot }}=-1, \mu=9+1.54 i, C_{1}=0.29, C_{2}=0.06$ and $t$ runs from 0 to 120 in steps of 0.1 .

Now we suppose that the rotating wave undergoes a Hopf bifurcation to a modulated rotating wave so $\lambda>0$ in the center bundle equations. Recall that the modulated rotating wave $u(t)$ admits the decomposition $u(t)=\gamma(t) y(t)$ where $\gamma(t) \in \Gamma$ and $y(t)$ is the solution for the normal vector field. Write $y(t)=v_{0}+w(t)$. For simplicity, we suppose that $w(t)$ is itself a pure Fourier-Bessel mode, proportional to $\operatorname{Im}\left(c J_{1}(\xi r) e^{i \theta}\right)$, where $c, \xi \in \mathbf{C}$. (In practice $w(t)$ will involve infinitely many such modes.) The amplitude and time dependence of $w(t)$ is determined by the shape variable $q(t)$ on the center bundle. Hence, we have

$$
w(t)=\operatorname{Im}\left(\sqrt{\lambda} c e^{i \omega_{\mathrm{bif}} t} J_{1}(\xi r) e^{i \theta}\right)
$$

In particular, the shape change of the modulated rotating wave is governed by

$$
y(t)=\operatorname{Im}\left(J_{1}(\mu r) e^{i \theta}+\sqrt{\lambda} c e^{i \omega_{\mathrm{bif}} t} J_{1}(\xi r) e^{i \theta}\right) .
$$


Thus in Figure 6(b) we plot the spiral tip for the function

$$
u(t)=\gamma(t) y(t)=R_{\varphi(t)} y(t)=\operatorname{Im}\left(\left(J_{1}(\mu r)+\sqrt{\lambda} c e^{i \omega_{\mathrm{bif}} t} J_{1}(\xi r)\right) e^{i\left(\theta-\omega_{\mathrm{rot}} t-\frac{\sqrt{\lambda}}{\omega_{\mathrm{bif}}} \sin \omega_{\mathrm{bif}} t\right)}\right),
$$

where $\omega_{\text {rot }}, \mu, C_{1}, C_{2}, t$ are as in Figure $6(\mathrm{a}), \sqrt{\lambda}=0.02, c=0.85-0.54 i, \omega_{\text {bif }}=-0.715$, $\xi=12-0.72 i$. The result is a 'fattening-out' of the perfect circle of Figure 6(a). In particular, there is no visible sign of meandering. However, the motion around the circle is nonuniform as shown by the graph of angle plotted against time in Figure 7 . The parameter settings in Figure 7 are identical to those in Figure 6(b), but with $t$ running from 0 to 12 in steps of 0.01 .

Figure 6(c)-(f) shows the spiral tip for functions of the form (7.1) subject to the time dependent planar translation

$$
z(t)=z-p(t)=z-\frac{\sqrt{\lambda}}{i\left(\omega_{\mathrm{rot}}+\omega_{\mathrm{bif}}\right)} e^{i\left(\omega_{\mathrm{rot}}+\omega_{\mathrm{bif}}\right) t}
$$

The parameters in Figures 6(b) and 6(c) are identical and it is evident that it is the additional translation modulation in (c) which is responsible for meandering of the spiral tip. Figures $6(d)$ and $6(\mathrm{e})$ show meandering before $\left(\omega_{\text {bif }}=0.895\right)$ and after $\left(\omega_{\text {bif }}=1.105\right)$ resonance and illustrate the transition from petals inwards to petals outwards. We choose $\omega_{\text {bif }}=1.005$ in (f) to obtain almost linear drift very close to resonance (in this last diagram, $t$ runs from 0 to 43 in steps of 0.1 ).

Figures 4 and 5 show the motion of the inner and outer patterns corresponding to the parameter settings in Figures $6(\mathrm{~b})$ and (c), but we have taken $\sqrt{\lambda}=0.2$ (instead of $\sqrt{\lambda}=$ 0.02 ) so that the contours corresponding to the inner and outer patterns can be distinguished.

\section{Completion of Proofs}

In this section, we verify the exponents of $\lambda$ given in Lemma 4.3, Lemma 4.4, Proposition 5.3 and Proposition 5.4.

Proposition 8.1 Consider the $O D E$

$$
\dot{q}=F^{q}(q, \lambda),
$$

where $F^{q}: \mathbf{R}^{2} \times \mathbf{R} \rightarrow \mathbf{R}^{2}$ is a general smooth vector field satisfying $F(0,0)=0$ and undergoing Hopf bifurcation with eigenvalues $\pm i \omega_{\mathrm{bif}}$. The branch of periodic solutions guaranteed 
by the Hopf Theorem can be written as a Fourier series

$$
q(t, \lambda)=\sum_{j \in \mathbf{Z}} q_{j}(\lambda) e^{i j \omega_{\mathrm{bif}}(\lambda) t}
$$

where $\omega_{\mathrm{bif}}(\lambda)$ is smooth in $\lambda, \omega_{\mathrm{bif}}(0)=\omega_{\mathrm{bif}}$. Moreover, generically the Fourier coefficients satisfy

$$
q_{0} \sim \lambda, \quad q_{j} \sim \lambda^{|j| / 2}, j \neq 0 .
$$

Proof If follows from Birkhoff normal form theory that there is a polynomial change of coordinates $q=P(z, \bar{z}, \lambda)$, where $P(0,0)=0$, such that the ODE $\dot{q}=F^{q}(q, \lambda)$ is transformed up to any finite order into the simpler ODE

$$
\dot{z}=g\left(|z|^{2}, \lambda\right) z
$$

where $g: \mathbf{R}^{2} \rightarrow \mathbf{R}$ is smooth. In these coordinates, the bifurcating periodic solutions are given by

$$
z(t, \lambda)=a(\lambda) e^{i \omega_{\mathrm{bif}}(\lambda) t}
$$

where $a(\lambda) \sim \lambda^{1 / 2}$. Transforming back into the original coordinates, we see that the linear terms in $P$ lead to the Fourier modes $j= \pm 1$ and generically $q_{ \pm 1} \sim \lambda^{1 / 2}$. Similarly, the quadratic interactions in $P$ contribute the Fourier modes $j=0$ and $j= \pm 2$ so that generically $q_{0}, q_{ \pm 2} \sim \lambda$. The Fourier modes $q_{ \pm j}, j \geq 3$ arise from the $j$ 'th order terms in $P$, so that $q_{ \pm j} \sim \lambda^{j / 2}, j \geq 3$.

Proposition 8.2 Assume the set up of Proposition 8.1, except that $F^{q}$ is a general smooth $\mathbf{Z}_{k}$-equivariant vector field on $\mathbf{R}^{2}$, where the action of $\mathbf{Z}_{k}$ on $\mathbf{R}^{2} \cong \mathbf{C}$ is given by

$$
R_{2 \pi / k} q=e^{2 \pi i m / k} q
$$

for some $m=1, \ldots,[k / 2]$. Let $d=\operatorname{gcd}(k, m)$ and $\ell=k / d$. The branch of periodic solutions guaranteed by the Hopf Theorem can be written as a Fourier series

$$
q(t, \lambda)=\sum_{s \in \mathbf{Z}} r_{s}(\lambda) e^{i(1+s \ell) \omega_{\mathrm{bif}}(\lambda) t}
$$

Moreover, generically the Fourier coefficients satisfy

$$
r_{s} \sim \lambda^{|1+s \ell| / 2} .
$$


Proof The spatiotemporal symmetry (4.7) places restrictions on the Fourier series in Proposition 8.1. Indeed, we calculate that if $q_{j} \not \equiv 0$ then

$$
e^{2 \pi i j / \ell}=e^{i j \omega_{\mathrm{bif}}(\lambda) T(\lambda) / \ell}=e^{2 \pi i / \ell}
$$

It follows that $j \equiv 1 \bmod \ell$. Hence, we may write

$$
q(t, \lambda)=\sum_{s \in \mathbf{Z}} r_{s}(\lambda) e^{i(1+s \ell) \omega_{\mathrm{bif}}(\lambda) t}
$$

and it follows from Proposition 8.1 that $r_{s}=O\left(\lambda^{|1+s \ell| / 2}\right)$.

It remains to verify that generically $r_{s}$ has terms of order precisely $|1+s \ell| / 2$ in $\lambda$. The reduction to Birkhoff normal form can be achieved by a $\mathbf{Z}_{k}$-equivariant change of coordinates $P$. We have the expansion

$$
q=P(z, \bar{z}, \lambda)=\sum a_{\mu, \nu}(\lambda) z^{\mu} \bar{z}^{\nu}
$$

and the $\mathbf{Z}_{k}$-equivariance condition $P\left(e^{2 \pi i m / k} z, e^{-2 \pi i m / k} \bar{z}, \lambda\right)=e^{2 \pi i m / k} P(z, \bar{z}, \lambda)$ implies that

$$
a_{\mu, \nu}(\lambda) e^{2 \pi i(\mu-\nu-1) m / k} \equiv 0
$$

Hence, either $a_{\mu, \nu} \equiv 0$ or

$$
(\mu-\nu-1) \frac{m / d}{\ell}=(\mu-\nu-1) \frac{m}{k} \in \mathbf{Z}
$$

Since $m / d$ and $\ell=k / d$ have no factors in common, we deduce that $\mu-\nu-1$ is a multiple of $\ell$. It follows that the surviving terms in $P$ have the form

$$
|z|^{2 a} z^{b \ell+1}, a, b \geq 0, \quad \text { and }|z|^{2 a} \bar{z}^{b \ell-1}, a \geq 0, b>0 .
$$

These terms yield Fourier coefficients

$$
q_{b \ell+1} \sim \lambda^{(b \ell+1) / 2}, b \geq 0, \quad \text { and } \quad q_{-(b \ell-1)} \sim \lambda^{(b \ell-1) / 2}, b>0
$$

respectively.

Proof of Lemma 4.3 The function $F^{\varphi}$ is a general smooth function of $q$ and $\lambda$ subject to the invariance condition (4.6). A computation similar to that for the function $P$ in the proof of Proposition 8.2 shows that the general term in $F^{\varphi}$ has the form $|q|^{2 a} q^{b \ell}$ or $|q|^{2 a} \bar{q}^{b \ell}$ where $a, b \geq 0$. 
In particular, the lowest order terms in $F^{\varphi}(q(t, \lambda), \lambda)$ correspond to $a=0, b=1$ and to $a=1, b=0$. Taking $a=0, b=1$, and substituting in the Fourier series (8.1) for $q$, yields lowest order terms of the form

$$
\left(r_{0}(\lambda) e^{i \omega_{\mathrm{bif}}(\lambda) t}\right)^{\ell}
$$

which is of the required order $\lambda^{\ell / 2}$.

It remains to show that the nonconstant terms produced by taking $a=1, b=0$ are of order at least $\lambda^{\ell / 2}$. By Proposition 8.2,

$$
|q(t, \lambda)|^{2}=\sum_{s_{1}, s_{2} \in \mathbf{Z}} r_{s_{1}}(\lambda) \bar{r}_{s_{2}}(\lambda) e^{i\left(s_{1}-s_{2}\right) \ell \omega_{\mathrm{bif}}(\lambda) t}
$$

Nonconstant terms are obtained when $s_{1} \neq s_{2}$. To obtain the smallest order in $\lambda$, we take $s_{1}=0, s_{2}=1$ which yields a term of order $\lambda^{1 / 2} \lambda^{(\ell-1) / 2}=\lambda^{\ell / 2}$. This completes the proof that $\lambda^{\ell / 2}$ is the lowest order nonconstant term in $F^{\varphi}$.

Proof of Lemma 4.4 In addition to the symmetry condition (4.6), which was taken into account in the proof of Lemma 4.3, the function $F^{\varphi}$ satisfies the condition $F^{\varphi}(\bar{q}, \lambda)=$ $-F^{\varphi}(q, \lambda)$. It follows that the general term in $F^{\varphi}$ has the form $|q|^{2 a}\left(q^{b \ell}-\bar{q}^{b \ell}\right)$ where $a, b \geq 0$.

It is immediate from the proof of Lemma 4.3 that no terms of order lower than $\lambda^{\ell / 2}$ are possible. The lowest order terms are obtained by taking $a=0$ and $b=1$, and this yields terms of order $\lambda^{\ell / 2}$ as before.

It remains to verify the order of the constant term in the Fourier expansion of $F^{\varphi}$. Substituting the Fourier series (8.1) into $q^{\ell}$ yields a term of the form

$$
\left(r_{0} e^{i \omega_{\mathrm{bif}} t}\right)^{\ell-1} r_{-1} e^{i(-\ell+1) \omega_{\mathrm{bif}} t}=r_{0}^{\ell-1} r_{-1},
$$

which has the required order $\frac{1}{2}(\ell-1)+\frac{|-\ell+1|}{2}=\ell-1$ in $\lambda$. Note also that $r_{0}$ and $r_{-1}$ are the lowest and second lowest order Fourier coefficients of $q$, so the only term of lower order term in $F^{\varphi}$ is the nonconstant term of order $\ell / 2$ that we computed earlier. Hence, the lowest order constant term is of order $\lambda^{\ell-1}$ as required.

Proof of Proposition 5.3 The structure of the $\dot{p}$ component of the skew product equations is as given prior to the statement of Proposition 5.3. A calculation as in Lemma 4.3 shows that the general terms in the Taylor expansion of $f(q, \lambda)$ have the form $|q|^{2 a} q^{b}$ and $|q|^{2 a} \bar{q}^{c}$ where $a, b, c \geq 0$ and $b m \equiv 1 \bmod k, c m \equiv-1 \bmod k$. The lowest order term is either $q^{\beta}$ or $\bar{q}^{\beta}$ (or possibly both). Since Hopf bifurcation occurs with exponent $\lambda^{1 / 2}$, we obtain the required exponent $\lambda^{\beta / 2}$ for $g$. This completes the verification of the claim.

Proof of Proposition 5.4 The exponent $|n| / 2$ is verified as follows. By taking $b=n \geq 1$ or $c=-n \geq 1$ in the proof of Proposition 5.3, and substituting in the Fourier expansion (8.1) 
of $q$, we obtain a term in $g$ proportional to $\left(\sqrt{\lambda} e^{i \omega_{\text {bif }}(\lambda) t}\right)^{n}$ (arising from the $s=0$ term in $q)$. This resonance term shows that the exponent $|n| / 2$ is achieved generically. Observe also that every multiple $r$ of the basic frequency $\omega_{\text {bif }}(\lambda)$ in $q$ occurs with the identical power $r$ of $\sqrt{\lambda}$. Hence $|n| / 2$ is the smallest possible exponent.

\section{Acknowledgments}

We wish to thank Dwight Barkley, Mike Field, Michael Gorman, Gemunu Gunaratne, Greg King, Antonio Palacios, Kay Robbins and Claudia Wulff for helpful conversations. This research was supported in part by NSF Grant DMS-9704980 (MG, IM), the Texas Advanced Research Program (003652037) (MG), and an NSERC Grant (VGL).

\section{References}

[1] C.D. Andereck, S.S. Liu and H.L. Swinney. Flow regimes in a circular Couette system with independently rotating cylinders. J. Fluid Mech. 164 (1986) 155-183.

[2] D. Barkley. A model for fast computer-simulation of waves in excitable media. Physica D 49 (1991) 61-70.

[3] D. Barkley. Euclidean symmetry and the dynamics of rotating spiral waves. Phys. Rev. Lett. 72 (1994) 165-167.

[4] D. Barkley, M. Kness and L. S. Tuckerman. Spiral-wave dynamics in a simple model of excitable media: The transition from simple to compound rotation. Phys. Rev. A 42 (1990) 2489-2492.

[5] A. Bayliss and B.J. Matkowsky. Nonlinear dynamics of cellular flames. SIAM J. Appl. Math. 52 (1992) 396-415.

[6] A. Bayliss, B.J. Matkowsky and H. Riecke. Structure and dynamics of modulated traveling waves in cellular flames. Physica D 74 (1994) 1-23.

[7] A. Bayliss, B.J. Matkowsky and H. Riecke. Symmetries in modulated traveling waves in combustion: jumping ponies on a merry-go-round. Pattern Formation: Symmetry Methods and Applications (J. Chadam and W. Langford eds.) Fields Institute Communications 5, AMS, 1995, 19-43. 
[8] P. Chossat and G. Iooss. Primary and secondary bifurcations in the Couette-Taylor problem. Japan J. Appl. Math. 2 No. 1 (1985) 37-68.

[9] K.T. Coughlin and P.S. Marcus. Modulated waves in Taylor-Couette flow. Part 1. Analysis J. Fluid Mech. 234 (1992) 1-18. Part 2. Numerical simulation. 19-46.

[10] M. Dellnitz, M. Golubitsky, A. Hohmann and I. Stewart. Spirals in scalar reaction diffusion equations, Int. J. Bifur. \& Chaos 5(6) (1995) 1487-1501.

[11] B. Fiedler, B. Sandstede, A. Scheel and C. Wulff. Bifurcation from relative equilibria of noncompact group actions: skew products, meanders and drifts. Documenta Math. 1 (1996) 479-505.

[12] B. Fiedler and D. Turaev. Normal forms, resonances, and meandering tip motions near relative equilibria of Euclidean group actions. Arch. Rational Mech. \&5 Anal. 145 (1998) $129-159$.

[13] M. Golubitsky, V.G. LeBlanc and I. Melbourne. Meandering of the spiral tip: an alternative approach. J. Nonlin. Sci. 7 (1997) 557-586.

[14] M. Golubitsky and I.N. Stewart. Hopf bifurcation in the presence of symmetry. Arch. Rational Mech. \& Anal. 87 No. 2 (1985) 107-165.

[15] M. Golubitsky, I. Stewart and D. Schaeffer. Singularities and Groups in Bifurcation Theory: Vol. II, Applied Mathematical Sciences 69, Springer-Verlag, New York, 1988.

[16] M. Gorman. Private communication.

[17] M. Gorman, M. el-Hamdi and K.A. Robbins. Hopping motion in ordered states of cellular flames. Combustion Sci. and Technology. 98 (1994) 25-35.

[18] M. Gorman, M. el-Hamdi and K.A. Robbins. Ratcheting motion of concentric rings in cellular flames. Phys. Rev. Lett. 76 (1996) 228-231.

[19] M. Gorman, C.F. Hamill, M. el-Hamdi and K.A. Robbins. Rotating and modulated rotating states of cellular flames. Combustion Sci. and Technology. 98 (1994) 25-35.

[20] M. Gorman and H.L. Swinney. Visual observation of the second characteristic mode in a quasiperiodic flow. Phys. Rev. Lett. 43 (1980) 1871-1875.

[21] M. Gorman and H.L. Swinney. Spatial and temporal characteristics of modulated waves in the circular Couette system. J. Fluid Mech. 117 (1982) 123-142. 
[22] M. Gorman, H.L. Swinney and D. Rand. Doubly periodic circular Couette flow: experiments compared with predictions from dynamics and symmetry. Phys. Rev. Lett 46 (1981) 992-995.

[23] G.H. Gunaratne, M. el-Hamdi, M. Gorman and K.A. Robbins. Asymmetric cells and rotating rings in cellular flames. Mod. Phys. Lett. B 10 (1996) 1379-1387.

[24] W. Jahnke, W.E. Skaggs and A.T. Winfree. Chemical vortex dynamics in the BelousovZhabotinsky reaction and in the two-variable Oregonator model. J. Phys. Chem. 93 (1989) 740-749.

[25] W. Jahnke and A.T. Winfree. A survey of spiral-wave behaviors in the Oregonator model. Int. J. Bifur. \& Chaos 1 (1991) 445-466.

[26] R. Kapral and K. Showalter. Chemical Waves and Patterns. Kluwer Academic Publishers, Amsterdam, 1995.

[27] M. Krupa. Bifurcations of relative equilibria. SIAM J. Math. Anal. 21 (1990) 1453-1486.

[28] A.S. Landsberg and E. Knobloch. Direction-reversing traveling waves. Phys. Lett. A 159 (1991) 17-20.

[29] G. Li, Q. Ouyang, V. Petrov and H.L. Swinney. Transition from simple rotating chemical spirals to meandering and traveling spirals. Phys. Rev. Lett. 77 (1996) 2105-2108.

[30] E. Lugosi. Analysis of meandering in Zykov kinetics. Physica D 40 (1989) 331-337.

[31] P.S. Marcus. Simulation of Taylor-Couette flow. Part 2. Numerical results for wavyvortex flow with one travelling wave. J. Fluid Mech. 146 (1984) 65-113.

[32] A. Palacios, M. Gorman and G.H. Gunaratne. Modal decomposition of hopping states in cellular flames. Chaos 9 (1999) 755-767.

[33] A. Palacios, G.H. Gunaratne, M. Gorman and K.A. Robbins. Cellular pattern formation in circular domains. Chaos 7 (3) (1997) 463-475.

[34] B.B. Plapp and E. Bodenschatz. Core dynamics of multi-armed spirals in RayleighBénard convection. Physica Scripta 67 (1996) 111-117.

[35] D. Rand. Dynamics and symmetry. Predictions for modulated waves in rotating fluids. Arch. Rational Mech. \&3 Anal. 79 (1982) 1-38. 
[36] M. Renardy. Bifurcation from rotating waves. Arch. Rational Mech. \& Anal. 79 (1982) 49-84.

[37] B. Sandstede, A. Scheel and C. Wulff. Center-manifold reduction for spiral waves. $C$. R. Acad. Sci., Serié I 324 (1997), 153-158.

[38] B. Sandstede, A. Scheel and C. Wulff. Dynamics of spiral waves in unbounded domains using center-manifold reductions. J. Diff. Eqns. 141 (1997) 122-149.

[39] G.S. Skinner and H.L. Swinney. Periodic to quasiperiodic transition of chemical spiral rotation. Physica D 48 (1991) 1-16.

[40] G.I. Taylor. Stability of a viscous liquid contained between two rotating cylinders. Phil. Trans. Roy. Soc. London A 223 (1923) 289-343.

[41] A.T. Winfree. The Geometry of Biological Time. Biomathematics 8, Springer, New York, 1980.

[42] C. Wulff. Theory of Meandering and Drifting Spiral Waves in Reaction-Diffusion Systems. Freie Universität Berlin, Thesis, 1996.

[43] L.-H. Zhang and H.L. Swinney. Nonpropogating oscillatory modes in Couette-Taylor flow. Phys. Rev. A 31 (1985) 1006-1009.

[44] V.S. Zykov. Cycloidal circulation of spiral waves in an excitable medium. Biofizika 31 (1986) 862-865.

[45] V.S. Zykov. Kinematics of nonstationary circulation of spiral waves in an excitable medium. Biofizika 32 (1987) 337-340. 